\title{
COMPLETE ALGEBRAIC VECTOR FIELDS ON AFFINE SURFACES
}

\author{
SHULIM KALIMAN, FRANK KUTZSCHEBAUCH, AND MATTHIAS LEUENBERGER
}

\begin{abstract}
Let AAuthol $(X)$ be the subgroup of the group $\operatorname{Authol}_{\text {hol }}(X)$ of holomorphic automorphisms of a normal affine algebraic surface $X$ generated by elements of flows associated with complete algebraic vector fields. Our main result is a classification of all normal affine algebraic surfaces $X$ quasi-homogeneous under $\mathrm{AAut}_{\text {hol }}(X)$ in terms of the dual graphs of the boundaries $\bar{X} \backslash X$ of their SNC-completions $\bar{X}$.
\end{abstract}

\section{Contents}

1. Introduction

1.1. Motivation and general background

1.2. Complete algebraic vector fields and quasi-homogeneity by algebraically generated automorphisms

2. Existence of Riccati fibration

3. Preliminaries about dual graphs

4. $\mathbb{P}^{1}$-fibrations

5. On non-affine base of Riccati fibration 17

6. Rational first integral

7. Dual graphs of curves in rational surfaces

8. Proof of the necessity part of the Main Theorem

9. Proof of the sufficiency part of the Main Theorem

10. Homogeneity

References

\section{INTRODUCTION}

1.1. Motivation and general background. In the last decades affine algebraic varieties and Stein manifolds with big (infinite-dimensional) automorphism groups have been studied intensively. Several notions expressing the fact that the automorphisms group of a manifold is big have been proposed. Among the most important of them are (algebraic) density property and holomorphic flexibility with the former implying the latter. Both density property and holomorphic flexibility show that the manifold in question is an Oka-Forstnerič manifold. This important notion has also recently merged from the intensive studies around the homotopy principle which goes back to

2000 Mathematics Subject Classification: 14R20,32M17.

Key words: affine varieties, group actions, one parameter subgroups, transitivity.

This work was done during visits of the last two authors to the University of Miami and during a visit of the first author to the University of Bern. We thank these institutions for their hospitality.The work of the last two authors was partially supported by Schweizerischer Nationalfond grant 200021$140235 / 1$. 
the 1930's and has had an enormous impact on the development of Complex Analysis with a constantly growing number of applications (for definitions and more information we refer the reader to [15]). The newly emerged area of Holomorphic Elliptic Geometry is devoted to the study of these properties.

In spite of the large number of examples of such highly symmetric objects their classification and the exact relations between all mentioned properties remain unclear even in dimension 2. In particular, we do not know the description of Stein surfaces $X$ on which the group of holomorphic automorphisms $\operatorname{Aut}_{\text {hol }}(X)$ acts transitively. Since such transitivity is an automatic consequence of flexibility, its study is an important first step in our program of Finding the exact relations between all known properties from Holomorphic Elliptic Geometry for surfaces.

In the algebraic case the analogous question of algebraic transitivity is classical, it was "almost" answered in the papers of Gizatullin and Danilov [16], [18]. We need the following definition to formulate their result.

Definition 1.1. We call a normal Stein (resp. affine algebraic) surface $X$ quasihomogeneous with respect to a subgroup $G$ of the group of its holomorphic (resp. algebraic) automorphisms if the natural action of $G$ has an open orbit in $X$ whose complement is at most finite. A normal affine algebraic surface is called quasi-homogeneous (without any reference to a group) if it is quasi-homogeneous with respect to the group $\operatorname{Aut}_{\text {alg }}(X)$ of algebraic automorphisms ${ }^{1}$.

For convenience of the reader let us also recall the following

Definition 1.2. An SNC-completion (short for simple normal crossing) of a normal affine algebraic surface $X$ is a normal complete algebraic surface $\bar{X}$ such that all irreducible components of the boundary curve $D=\bar{X} \backslash X$ are smooth and all of its singularities are simple nodes (i.e., locally each of these singularities is a transversal intersection of two smooth analytic branches). The boundary $D$ is contained in the smooth part of the surface $\bar{X}$.

Every quasi-homogeneous surface is either the two-dimensional torus $\mathbb{C}^{*} \times \mathbb{C}^{*}$, or $\mathbb{C} \times \mathbb{C}^{*}$ or it admits an SNC-completion $\bar{X}$ such that the dual graph $\Gamma$ of its boundary $\bar{X} \backslash X$ (see Definition 3.1) is a linear rational graph [16], [18] which can be always chosen in the following standard form (so-called standard zigzag)

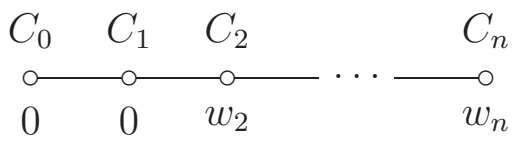

where $n \geq 1$ and $w_{i} \leq-2$ for $i=2, \ldots, n$. A surface that admits such a completion

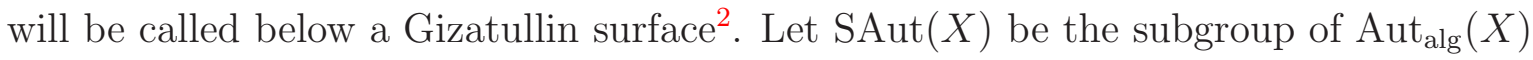

\footnotetext{
${ }^{1}$ When the complement to the open orbit is empty one has transitivity. However, there are examples of smooth quasi-homogeneous surfaces for which the complements of the open orbits are not empty. In the case of surfaces over algebraically closed field of positive characteristic they appeared already in the paper of Gizatullin and Danilov [17] who also knew but did not publish such examples for characteristic zero. In a published form examples of complex quasi-homogeneous surfaces with non-empty complements can be found in a recent paper of Kovalenko [26].

${ }^{2}$ In fact, for a normal affine surface to be a Gizatullin one it suffices to require that the dual graph of the boundary divisor is contractible to a linear graph. The class of Gizatullin surfaces coincides
} 
generated by elements of $\mathbb{G}_{a}$-actions ${ }^{3}$ (also called algebraic $\mathbb{C}_{+}$actions). Then this subgroup possesses already an open orbit in $X$ whose complement is at most finite. Recall that a $\mathbb{G}_{a}$-action can be viewed as the flow of a complete algebraic vector field on $X$ that is locally nilpotent.

More precisely, a holomorphic vector field $\nu$ on a complex space $X$ is called complete if the solution of the ODE

$$
\frac{d}{d t} \varphi(x, t)=\nu(\varphi(x, t)) \quad \varphi(x, 0)=x
$$

is defined for all complex times $t \in \mathbb{C}$ and all initial values $x \in X$. The induced maps $\Phi_{t}: X \rightarrow X$ given by $\Phi_{t}(x)=\varphi(x, t)$ yield the flow of $\nu$ which is nothing but a one-parameter subgroup in the group $\operatorname{Aut}_{\text {hol }}(X)$ of holomorphic automorphism with parameter $t \in \mathbb{C}_{+}$(equivalently, a holomorphic $\mathbb{C}_{+}$-action).

One could wish to extend the quasi-homogeneity results to the analytic situation replacing locally nilpotent vector fields by complete holomorphic vector fields and $\operatorname{SAut}(X)$ by its holomorphic analogue, but unfortunately the classification of complete holomorphic vector fields on Stein surfaces with sufficiently big automorphism groups (even on $\mathbb{C}^{2}$ ) seems still far out of reach. However, complete algebraic vector fields have been classified on $\mathbb{C}^{*} \times \mathbb{C}^{*}\left(\right.$ actually on $\left.\left(\mathbb{C}^{*}\right)^{n}\right)$ by Andersén using Nevanlinna theory [1] and on $\mathbb{C}^{2}$ by Brunella [8] using foliation theory. It is important to remember that when $X$ is an affine algebraic variety and $\nu$ is a complete algebraic vector field its flow may well be non-algebraic. When the flow is algebraic then either $\nu$ is locally nilpotent and we have a $\mathbb{G}_{a}$-action, i.e., an algebraic $\mathbb{C}_{+}$-action, or $\nu$ is semi-simple and we have a $\mathbb{G}_{m}$-action, i.e., an algebraic $\mathbb{C}^{*}$-action. ${ }^{4}$

Definition 1.3. A holomorphic automorphism $\alpha$ of an algebraic variety $X$ will be called algebraically generated if $\alpha$ coincides with an element $\Phi_{t}$ of the flow of a complete algebraic vector field on $X$ as before. The subgroup of the holomorphic automorphism group generated by such algebraically generated automorphisms will be denoted by AAuthol $_{\text {h }}(X)$ and a normal affine algebraic surface $X$ quasi-homogeneous with respect to $\operatorname{AAut}_{\text {hol }}(X)$ will be called generalized Gizatullin surface. If a normal affine algebraic surface $Y$ admits two complete non-proportional algebraic vector fields $\nu_{1}$ and $\nu_{2}$ (i.e., $f_{1} \nu_{1} \neq f_{2} \nu_{2}$ for any pair of nonzero regular functions $f_{1}$ and $f_{2}$ on $Y$ ) then there is an open orbit of the natural AAut $_{\text {hol }}(Y)$-action in $Y$. In what follows we call such $Y$ a surface with an open orbit (without mentioning $\operatorname{AAut}_{\text {hol }}(Y)$ ). Of course, every generalized Gizatullin surface is a surface with an open orbit.

also with the class of surfaces with a trivial Makar-Limanov invariant, i.e., $\operatorname{ML}(X)=\mathbb{C}$ where $\operatorname{ML}(X)$ is the subring of the ring of regular functions on $X$ that consists of all functions invariant under any (algebraic) $\mathbb{G}_{a}$-action on $X$

${ }^{3}$ Recall that given a variety $V$ over a field $\mathbb{k}$ an algebraic action of the additive group $\mathbb{k}$ (resp. multiplicative group $\mathbb{k}^{*}=\mathbb{k} \backslash\{0\}$ ) on $V$ is called a $\mathbb{G}_{a}$-action (resp. $\mathbb{G}_{m}$-action.) In particular, in the case of $\mathbb{k}=\mathbb{C}$ a $\mathbb{G}_{a}$-action is the same as an algebraic action of the group $\mathbb{C}_{+}$of complex numbers with addition as the group operation.)

${ }^{4}$ It is worth reminding that an algebraic vector field $\nu$ on an affine algebraic variety $X$ is locally nilpotent if and only if for every element $f$ of the algebra $A$ of regular functions on $X$ there exists $n=n(f)$ such that $\nu^{n}(f)=0$. Similarly, $\nu$ is semi-simple if and only if $A$ is a $\mathbb{Z}$-graded algebra $A=\bigoplus_{k \in \mathbb{Z}} A_{k}$ such that for every $f \in A_{k}$ one has $\nu(f)=k f$. 
It is worth mentioning that the first examples of generalized Gizatullin surfaces with discrete algebraic automorphism group $\operatorname{Aut}_{\text {alg }}(X)$ were found by the first and second author in [23], [24] and they can be presented as hypersurfaces $\{x p(x)+y q(y)+x y z=$ $1\} \subset \mathbb{C}_{x, y, z}^{3}$ where the polynomials $1-x p(x)$ and $1-y q(y)$ have simple roots only (none of these surfaces admits nontrivial algebraic $\mathbb{G}_{a}$ or $\mathbb{G}_{m}$-actions). Similar to the case of the torus for each such a surface $X$ an SNC-completion $\bar{X}$ can be chosen as a cycle.

In this paper we deal with the following first step of our general program.

\subsection{Complete algebraic vector fields and quasi-homogeneity by algebraically} generated automorphisms. The main result of our paper is the following classification of normal affine algebraic surfaces quasi-homogeneous under the group of algebraically generated automorphisms.

Theorem 1.4. A normal complex affine algebraic surface $X$ is generalized Gizatullin if and only if it admits an SNC-completion $\bar{X}$ for which the boundary $\bar{X} \backslash X$ is connected, consists of rational curves, and has a dual graph $\Gamma$ that belongs to one of the following types

(1) a standard zigzag or a linear chain of three 0-vertices (i.e., Gizatullin surfaces and $\mathbb{C} \times \mathbb{C}^{*}$,

(2) circular graph with the following possibilities for weights

$$
\left(\left(0,0, w_{1}, \ldots, w_{n}\right)\right) \text { where } n \geq 0 \text { and } w_{i} \leq-2,
$$

$$
((0,0, w)) \text { with }-1 \leq w \leq 0 \text { or }((0,0,0, w)) \text { with } w \leq 0
$$

(3)

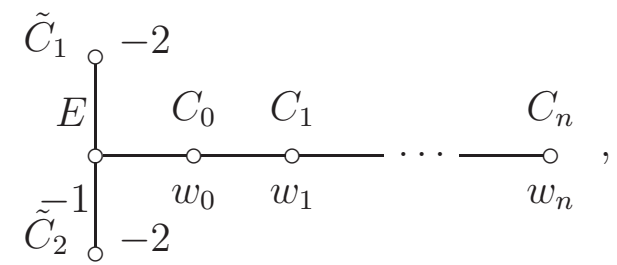

(4)
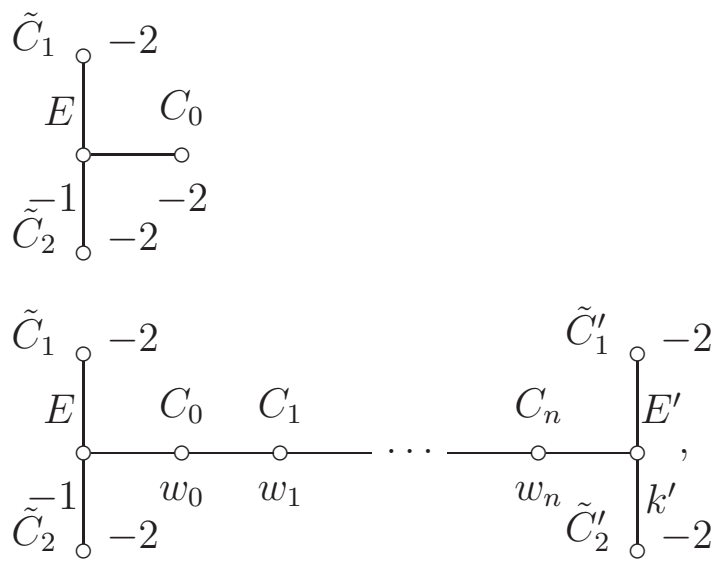


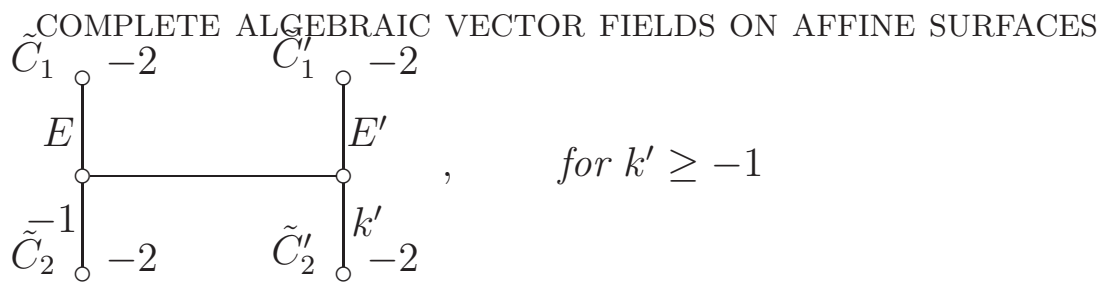

where $n \geq 0, w_{i} \leq-2$ for $i \geq 1$ and

$(\alpha)$ in (3) either $n=0$ and $0 \leq w_{0} \leq 2$ or $n \geq 1$ and $0 \leq w_{0} \leq 1$;

$(\beta)$ in (5) either $n=w_{0}=0$ and $k^{\prime} \leq-1$ or $n \geq 1,0 \leq w_{0} \leq 1$ and $k^{\prime} \leq-3$ or $n \geq 1,0 \leq w_{0} \leq 1, w_{n} \leq-3$ and $k^{\prime}=-2$;

$(\gamma)$ in (2a) $\Gamma$ is a subgraph of a graph contractible to the cycle $((0,0,0,0))$.

It should be mentioned that the second author announced a formulation of Theorem 1.4 in the survey [25] but unfortunately in that formulation conditions $(\alpha)$ and $(\beta)$ were stated incorrectly which results in some extra graphs that do not appear as the dual graphs of SNC-curves in smooth complete surfaces.

In the framework of our general program Theorem 1.4 provides us with a list $^{5}$ of affine algebraic surfaces on which one can look for holomorphic flexibility, algebraic density property, or even classification of all complete algebraic vector fields. A case of particular surfaces has been already worked out by the third author in [28]. Following our general program the algebraic density property for certain classes of Gizatullin surfaces has been recently established by Andrist, Poloni and the second author in [3] and by Andrist in [2].

The second important result of our paper is one of the essential ingredients in the proof of our main result.

Theorem 1.5. Let $X$ be a normal affine algebraic surface which admits a nonzero complete algebraic vector field. Then either:

(1) all complete algebraic fields share the same rational first integral (i.e., there is a rational map $f: X \rightarrow B$ onto a smooth curve $B$ such that all complete algebraic vector fields on $X$ are tangent to the fibers of $f$ ), or

(2) $X$ is a rational surface with an open orbit and, furthermore, for every complete algebraic vector field $\nu$ on $X$ there is a regular function $f: X \rightarrow \mathbb{C}$ (depending on $\nu$ ) with general fibers isomorphic to $\mathbb{C}$ or $\mathbb{C}^{*}$ such that the flow of $\nu$ sends fibers of $f$ to fibers of $f$ and $f(X)$ is either $\mathbb{C}$ or $\mathbb{C}^{*}$.

The fact that the flow sends fibers to fibers can be reformulated as follows: there is a complete vector field $\nu_{0}$ on $\mathbb{C}$ such that $f$ is $\mathbb{C}_{+}$-equivariant with respect to the flows of $\nu$ (resp. $\left.\nu_{0}\right)$ acting on $X$ (resp. $\mathbb{C}$ ). When $\nu_{0}$ is trivial then $f$ is again a rational first integral of $\nu$ (which is regular in this case)

\footnotetext{
${ }^{5}$ It should be emphasized that in general two surfaces with the same boundary graph as one of those which appear in Theorem 1.4 are not necessarily homeomorphic. Even in the case of the same topology such surfaces may admit families with non-isomorphic members (we do not know if a similar fact holds in the analytic setting). Furthermore, homogeneity of such surfaces with respect

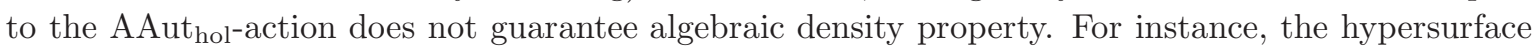
$\{x p(x)+y q(y)+x y z=1\} \subset \mathbb{C}_{x, y, z}^{3}$ mentioned before does not have it, though it has another positive feature - the algebraic volume density property [24].
} 
In the special case of $X=\mathbb{C}^{2}$ Theorem 1.5 was proven by Brunella [8] and our original proof followed his approach (see Remark 2.14). However, after finishing the first version of the manuscript we were informed about the paper of Guillot and Rebelo [20]. They proved a very general analogue of Theorem 1.5 for so called semicomplete meromorphic vector fields on complex surfaces in which the conclusion about the isomorphism type of a general fiber of $f$ in (2) is replaced by the following: the completion of such a fiber is either a rational curve or an elliptic one. Complete algebraic fields on affine algebraic surfaces are, of course, semicomplete, and it is easier to extract Theorem 1.5 from the Guillot-Rebelo result which we do in the present version.

The paper is organized as follows. In Section 2 we start the proof of a crucial part, the case of absence of a rational first integral, of Theorem 1.5 based on the Guillot-Rebelo theorem.

In Section 3 we remind some facts from [11] (see also [9], [10]) about weighted dual graphs of algebraic curves contained in smooth surfaces while in Section 4 we present some basic results on $\mathbb{P}^{1}$-fibrations on $\bar{X}$ extending $\mathbb{C}$ - or $\mathbb{C}^{*}$-fibrations on $X$.

In Section 5, with these preliminaries in hand we can finish the case of absence of a rational first integral from Theorem 1.5.

Section 6 is devoted to the geometrical description of rational first integrals of complete algebraic vector fields on smooth semi-affine surfaces (whenever such integrals exist) which together with the results from Section 2 and Section 5 allows us to complete the proof of Theorem 1.5.

In Section 7 we show that the graphs listed in Theorem 1.4 appear as the dual graphs of SNC-curves with rational irreducible components contained in smooth rational surfaces.

In Section 8 we show there is no graph different from those described in (1)-(6) that can serve as the dual graph of a boundary of a surfaces quasi-homogeneous under $\operatorname{AAut}_{\text {hol }}(X)$.

In Section 9 we establish that any surface with a boundary graph as in (1)-(6) is indeed quasi-homogeneous under $\operatorname{AAut}_{\text {hol }}(X)$ which concludes the proof of Theorem 1.4 .

In the last Section we show that some of the surfaces in Theorem 1.4 are in fact AAut $_{\text {hol }}(X)$-homogeneous. In particular, this class includes all surfaces of type (2) and all known Gizatullin surfaces that are not homogeneous with respect to the natural $\operatorname{Aut}(X)$-action described in [26].

\section{Existence of RicCATi fibration}

Definition 2.1. A semi-affine surface is an algebraic surface $X$ that admits a proper birational morphism onto an affine algebraic surface $S$ (which in complex analysis is nothing but the Remmert reduction). For instance, resolution of singularities of a normal affine surface $S$ leads, of course, to a smooth semi-affine surface $X$.

Remark 2.2. Note that the boundary divisor $D=\bar{X} \backslash X$ for some SNC-completion $\bar{X}$ of $X$ can be also viewed as the boundary divisor of some SNC-completion $\bar{S}$ of $S$ and the intersection matrix of the irreducible components of $D$ in $\bar{X}$ is the same as the similar matrix for $D$ in $\bar{S}$. 
We shall use the following standard fact.

Proposition 2.3. Algebraic vector fields on $S$ lift to algebraic vector fields on $X$ and complete ones lift to complete ones. Vice versa, complete vector fields on $X$ correspond to complete vector fields on $S$.

Proof. For simplicity let us deal with complete algebraic fields only. Consider the flow of such a field. The singularities of $S$ are fixed by this flow. Hence, it induces a holomorphic $\mathbb{C}_{+}$-action on the tangent cones at these points, and, therefore, on the result $\tilde{S}$ of blowing $S$ up at these singularities (since the exceptional curves in $\tilde{S}$ are projectivizations of tangent cones). Then the action on $\tilde{S}$ induces a holomorphic $\mathbb{C}_{+^{-}}$ action on its normalization. Since a minimal resolution of surface singularities involves only a sequence of blowing-up and normalizations [29] we get a holomorphic $\mathbb{C}_{+}$-action on $X$ and, thus, the desired complete algebraic vector field on $X$.

Vice versa, given a complete algebraic vector field on $X$ we note that its flow preserves complete curves contained in $X$ since the number of such curves in a semi-affine surface is finite. Thus, one can push this flow down to the regular part of $S$ and extend the holomorphic $\mathbb{C}_{+}$-action to $S$ by Hartogs' theorem. This yields a complete algebraic vector field on $S$.

Therefore, to consider complete algebraic vector fields on normal affine surfaces is the same as to consider such fields on smooth semi-affine surfaces.

For convenience of the reader we recall the following

Definition 2.4. A foliation $\mathcal{F}$ on a smooth complex surface $\bar{X}$ is given by an open covering $\left\{U_{j}\right\}$ of $\bar{X}$ and holomorphic vector fields $\nu_{j} \in H^{0}\left(U_{j}, T \bar{X}\right)$ with isolated zeros such that

$$
\nu_{i}=g_{i j} \nu_{j} \text { on } U_{i} \cap U_{j}
$$

for invertible holomorphic functions $g_{i j} \in H^{0}\left(U_{i} \cap U_{j}, \mathcal{O}_{\bar{X}}^{*}\right)$ where $\mathcal{O}_{\bar{X}}^{*}$ is the sheaf of invertible functions. Gluing orbits of $\left\{\nu_{j}\right\}$ one gets leaves of the foliation $\mathcal{F}$. The singular set $\operatorname{Sing}(\mathcal{F})$ is the discrete subset of $\bar{X}$ whose intersection with each $U_{j}$ coincides with the zeros of $\nu_{j}$. The cocycle $\left\{g_{i j}\right\}$ defines a holomorphic line bundle $K_{\mathcal{F}}$ which is called the canonical bundle of the foliation $\mathcal{F}$.

One of the main facts about foliations which is of use for us is the following consequence of the Camacho-Sad formula (e.g., see [6]).

Proposition 2.5. Let $\mathcal{F}$ be a foliation on a compact smooth surface $\bar{X}$ and $C$ be an $\mathcal{F}$-invariant closed curve that does not contain singularities of $\mathcal{F}$. Then the selfintersection $C \cdot C$ of $C$ is zero.

Setting 2.6. Let $X$ be a smooth semi-affine smooth algebraic surface equipped with a nonzero complete algebraic vector field $\nu$ and an SNC-completion $\bar{X}$ (i.e., $\bar{D}=\bar{X} \backslash X$ is an SNC-divisor in the smooth projective surface $\bar{X}$ ). The algebraic vector field $\nu$ generates a rational vector field $\bar{\nu}$ on $\bar{X}$. Note also that $\nu$ generates a foliation $\mathcal{F}$ on $X$ which extends to the foliation $\overline{\mathcal{F}}$ on $\bar{X}$ generated by $\bar{\nu}$.

As we mentioned before, complete algebraic vector fields on $X$ form a subset in the wider class of semi-complete vector fields. Therefore, in our case Theorem B in [20] implies the following. 
Theorem 2.7. In Setting 2.6 either

(1) $\bar{\nu}$ has a first integral ${ }^{6}$, or

(2) up to a birational transformation of $\bar{X}$ the field $\bar{\nu}$ is holomorphic, or

(3) there is a morphism $f: \bar{X} \rightarrow B$ into a complete rational or elliptic curve $B$ with rational or elliptic general fibers such that for some vector field $\mu$ on $B$ one has $f_{*}(\bar{\nu})=\mu$ (the flow of $\bar{\nu}$ preserves the fibration $\left.f\right)$.

Definition 2.8. A morphism $f: \bar{X} \rightarrow B$ as above with rational (resp. elliptic) general fibers is called a Riccati (resp. Turbulent) fibration adapted to the foliation $\overline{\mathcal{F}}$.

Proposition 2.9. In Setting 2.6 let $X$ be a semi-affine smooth surface and $f: \bar{X} \rightarrow B$ be a fibration adapted to $\overline{\mathcal{F}}$ as in Theorem 2.7 (3). Suppose that $\bar{\nu}$ does not have a rational first integral. Then $f$ is a Riccati fibration and its restriction to $X$ yields a morphism with general fibers isomorphic either to $\mathbb{C}^{*}$ or to $\mathbb{C}$. Furthermore, $B$ is a rational curve.

Proof. Since $X$ is a semi-affine surface it may contain only a finite number of complete curves. Hence, a general fiber $F=f^{-1}\left(b_{0}\right)$ of $f$ (which is isomorphic by definition either to $\mathbb{P}^{1}$ or to a complete elliptic curve) must meet the union $C$ of the components of boundary divisor $D=\bar{X} \backslash X$ that are not contained in the fibers of $f$. The absence of first integrals implies that $\bar{\nu}$ is not tangent to $F$ but it is tangent to $C$ since $\nu$ is a complete field. In particular, $C$ consists of leaves of the foliation $\overline{\mathcal{F}}$.

Furthermore, $\mu$ from Theorem 2.7 (3) (which is complete by construction) must be nonzero. Hence, removing from $B$ a finite number of points corresponding to nongeneral values of $f$ we get a curve $B^{*}$ which is an orbit of the flow of $\mu$. Thus, if $B$ is rational then $B^{*}$ is isomorphic either to $\mathbb{C}$ or to $\mathbb{C}^{*}$ while in the case of an elliptic curve $B^{*}=B$ since the lift of a vector field $\mu$ (which is not identically zero) to the universal cover $\mathbb{C}$ of $B$ may be only a nonzero constant field, i.e., $\mu$ does not vanish at any point of $B$.

Note that foliation $\overline{\mathcal{F}}$ generates a representation of the fundamental group $\pi_{1}\left(B^{*}, b_{0}\right)$ into the group $\operatorname{Aut}_{\text {hol }}(F)$ of holomorphic automorphisms of $F$ which must preserve the finite nonempty subset $F \cap C$ (because the leaves of the foliation are invariant under monodromy).

To get a contradiction assume that the curve $F \backslash C$ is of general type (i.e., either $F$ is elliptic or $F \simeq \mathbb{P}^{1}$ and $F \cap C$ consists at least of three points). Then the image of $\pi_{1}\left(B^{*}, b_{0}\right)$ under the representation is contained in a finite $\operatorname{subgroup} G \simeq \operatorname{Aut}(F \backslash C)$ of $\operatorname{Aut}_{\text {hol }}(F)$. Consider the unramified cover $\tilde{B}^{*} \rightarrow B^{*}$ for which $G$ plays the role of the Galois group, the fibered product $\tilde{X}^{*}=X \times_{B} \tilde{B}^{*}$, the preimage $\tilde{C}^{*}$ of $C$ in $\tilde{X}^{*}$ and the natural projection $\tilde{f}: \tilde{X}^{*} \rightarrow \tilde{B}^{*}$. Note that every irreducible component of $\tilde{C}^{*}$ is now a section of $\tilde{f}$ and the vector field $\tilde{\nu}$ on $\tilde{X}^{*}$ induced by $\nu$ is tangent to $\tilde{C}^{*}$ and sends every fiber of $\tilde{f}$ to a fiber of $\tilde{f}$.

Claim. For the first statement it suffices to show that there is a Zariski dense open subset $U$ of $\tilde{B}^{*}$ and $F \backslash C \subset F^{*} \subset F$ such that (a) $F^{*}$ is a curve of general type and (b) $\tilde{f}^{-1}(U)$ is naturally isomorphic to $U \times F$ over $U$.

Indeed, then $\tilde{\nu}$ is tangent to the curve $U \times\left(F \backslash F^{*}\right) \subset \tilde{C}^{*}$ (i.e., the flow of $\tilde{\nu}$ transforms $U \times F^{*}$ into itself) and since $\tilde{f}_{*}(\tilde{\nu})$ is a vector field on $U$ and $F^{*}$ is of general type for

\footnotetext{
${ }^{6}$ I.e., a surjective rational map $\bar{X}-\rightarrow C$ into a curve $C$ whose fibers are tangent to $\bar{\nu}$.
} 
every element $U \times F^{*} \rightarrow U \times F^{*}$ of the flow the second coordinate function must yield the identity map $F^{*} \rightarrow F^{*}$. That is, the natural projection $\tau: \tilde{f}^{-1}(U) \rightarrow F$ is a rational first integral for $\tilde{\nu}$. Consider now a rational function $h$ on $F$ that has pole at every point of $F \cap C$. Let $h_{1}, \ldots, h_{m}$ be the orbit of $h$ under the natural action of $G$. Since $C \cap F$ is invariant under the action of $G$ we see that each $h_{i}$ has a pole at every point of $F \cap C$. In particular, the product $h_{1} \cdot \ldots \cdot h_{m}$ is a non constant $G$-invariant meromorphic function on $F$. Taking the composition of this product with $\tau$ one gets a rational function $\tilde{h}$ on $\tilde{X}^{*}$ invariant with respect to the natural $G$-action. Hence, $\tilde{h}$ induces a rational function on $\bar{X}$ which is a well-defined first integral of $\bar{\nu}$ contrary to the assumption. This concludes the proof of the Claim.

Let us now check conditions (a) and (b). If $F=\mathbb{P}^{1}$ then $\tilde{X}^{*}$ is a trivial $\mathbb{P}^{1}$-bundle over $\tilde{B}^{*}$ since $\tilde{B}^{*}$ is affine, i.e., we have (b) with $U=\tilde{B}^{*}$. Note that $\tilde{C}^{*}$ consists at least of three irreducible components. Choosing the isomorphism $\tilde{X}^{*} \simeq \tilde{B}^{*} \times \mathbb{P}^{1}$ appropriately one can suppose that these three sections can be viewed as the curves $\tilde{B}^{*} \times \infty, \tilde{B}^{*} \times 0$ and $\tilde{B}^{*} \times 1$ in $\tilde{B}^{*} \times \mathbb{P}^{1}$. Thus, letting $F^{*}=\mathbb{P}^{1} \backslash\{0,1, \infty\}$ we get (a) which concludes the proof of the first statement in the case of $F=\mathbb{P}^{1}$.

Now let us deal with the case when $F$ is elliptic. Consider the generic fiber of $\tilde{f}$. It is an elliptic curve $E$ over the field $\mathbb{K}$ of rational functions on $\tilde{B}^{*}$ and (being sections of $\tilde{f}$ ) the irreducible components of $\tilde{C}^{*}$ can be treated as closed points in $E$. Recall that $E$ can be viewed as a smooth cubic in $\mathbb{K} \mathbb{P}^{2}$ given by an equation in the Weierstrass form $\breve{y}^{2}=\breve{x}^{3}+a_{4} \breve{x} \breve{z}^{2}+a_{6} \breve{z}^{3}$ where $a_{4}, a_{6} \in \mathbb{K}$ and $(\breve{x}: \breve{y}: \breve{z})$ is a homogeneous coordinate system on $\mathbb{K P}^{2}$ (e.g., see [37]). In particular, $a_{4}=a_{4}(\tilde{b})$ and $a_{6}=a_{6}(\tilde{b})$ are rational functions on $\tilde{B}^{*}$. Since the flow of $\tilde{\nu}$ transforms the fibers of $\tilde{f}$ into the fibers of $\tilde{f}$ we see that for general $\tilde{b}_{0}$ and $\tilde{b}$ in $\tilde{B}^{*}$ the elliptic curves in $\mathbb{C P}^{2}$ given by the equations $\breve{y}^{2}=\breve{x}^{3}+a_{4}\left(\tilde{b}_{0}\right) \breve{x} \breve{z}^{2}+a_{6}\left(\tilde{b}_{0}\right) \breve{z}^{3}$ and $\breve{y}^{2}=\breve{x}^{3}+a_{4}(\tilde{b}) \breve{x} \breve{z}^{2}+a_{6}(\tilde{b}) \breve{z}^{3}$ are isomorphic. Hence they have the same $j$-invariant (e.g., see [37]) which implies that, fixing $\tilde{b}_{0}$ one has $a_{4}(\tilde{b})=a_{4}\left(\tilde{b}_{0}\right) u(\tilde{b})^{4}$ and $a_{6}(\tilde{b})=a_{6}\left(\tilde{b}_{0}\right) u(\tilde{b})^{6}$ where $u(\tilde{b})$ depends on $\tilde{b}$. Assuming for simplicity that $a_{4} \neq 0 \neq a_{6}$ we observe that $u^{2}(\tilde{b})$ is a rational function on $\tilde{B}^{*}$ and, thus, dividing by its powers we can suppose that for every $\tilde{b}$ in a nonempty Zariski open subset $U$ of $\tilde{B}^{*}$ the fiber $\tilde{f}^{-1}(\tilde{b})$ is given by an equation $\breve{y}^{2}=\breve{x}^{3}+a_{4} \breve{x}^{2}+a_{6} \breve{z}^{3}$ where $a_{4}$ and $a_{6}$ are now complex numbers (i.e., we have (b)). Since $E$ acts transitively on itself we can suppose that one of the irreducible components of $\tilde{C}^{*}$ corresponds to the point $(0: 0: 1)$ and that every fiber of $\tilde{f}^{-1}(\tilde{b}) \backslash \tilde{C}^{*}, \tilde{b} \in U$ is a curve contained in the affine curve $F^{*} \subset \mathbb{C}^{2}$ given by $y^{2}=x^{3}+a_{4} x+a_{6}$ which yields (a). Thus, we have the first statement.

If we assume that $B$ is elliptic then it was shown already that $\mu$ and, therefore, $\bar{\nu}$ do not vanish. That is, every irreducible component of $C$ has no singularities of $\overline{\mathcal{F}}$. This implies that these components are actually connected components of $C$ and furthermore $C$ coincides with the boundary divisor $D$ (indeed, otherwise $C$ contains singularities of $D$ which must be automatically singularities of $\overline{\mathcal{F}}$ ). By Proposition $2.5 D \cdot D=0$. However, by Remark $2.2 \mathrm{D}$ can serve also as a boundary of an SNC-completion of an affine surface. In particular, it is connected (by the Lefschetz hyperplane section theorem) and being a support of an ample divisor (Theorem 2 in [19]) it has a positive self-intersection number $D \cdot D>0$. This contradiction implies that $B$ cannot be elliptic and, thus, $B$ is rational. 
Remark 2.10. This argument showing that $F \backslash C$ cannot be hyperbolic essentially appeared already in the proof of $[8$, Lemma 1] in which Brunella showed that the foliation induced by a complete vector field on $\mathbb{C}^{2}$ cannot be a Turbulent one.

Corollary 2.11. Under the assumption of Proposition 2.9 the surface $X$ is also rational.

Proof. This follows from the fact that $B$ and general fibers of the morphism $f: \bar{X} \rightarrow B$ are rational.

Proposition 2.12. The case (2) of Theorem 2.7 occurs only if one of cases (1) or (3) occurs as well.

Proof. Suppose that $\psi: \bar{X}-\rightarrow Y$ is a birational map of $\bar{X}$ into a smooth projective surface $Y$ which transforms $\bar{\nu}$ into a holomorphic field $\mu$ on $Y$.

Note that the image $T$ of $D$ under $\psi$ cannot be a point. Indeed, by [22] both $\bar{X}$ and $Y$ can be obtained via contractions $\alpha: Z \rightarrow \bar{X}$ and $\beta: Z \rightarrow Y$ from the same smooth surface $Z$. Note that for $E=\alpha^{-1}(D)$ the surface $Z \backslash E$ is semi-affine since $\bar{X} \backslash D$ is. In particular, one has a birational morphism $\gamma: Z \backslash E \rightarrow S$ into an affine surface $S$. By construction the rational map $\gamma \circ \beta^{-1}: Y \backslash T-\rightarrow S$ is birational and, furthermore, it is regular by Hartogs' theorem. Thus, $Y \backslash T$ is semi-affine and $T$ is a divisor.

Claim. The Kodaira dimension of $Y$ cannot be non negative.

Assume the contrary. Then by [6, Ch. 6, Prop. 5] $\mu$ does not vanish and either it generates a foliation on $Y$ with elliptic curves as general leaves, or $Y$ is a torus and $\mu$ generates a Kronecker foliation (for definition see [6]) on it. The former case is impossible. Indeed, a general leave $G$ of the foliation $\mathcal{G}$ induced by $\mu$ contains an isomorphic image of an integral curve of $\nu$ under $\psi$ (because the rational map $\psi$ is regular outside codimension 2). Such an integral curve is automatically isomorphic to $\mathbb{C}$ or $\mathbb{C}^{*}$ since $\nu$ is a complete vector field on the semi-affine surface $X$. Thus, $G$ is rational and we need to consider the case of the Kronecker foliation only.

By definition any Kronecker foliation $\mathcal{G}$ has no algebraic curves among its leaves. Since $\mu$ is nowhere vanishing but every complete vector field on $\mathbb{P}^{1}$ has zeros we see that a general leaf $G$ of $\mathcal{G}$ is isomorphic to either $\mathbb{C}$ or $\mathbb{C}^{*}$. If $G \simeq \mathbb{C}^{*}$ then $G$ is an image of a general integral curve of $\nu$. Since these integral curves do not meet $D$ we conclude that $G$ does not meet $T$. This implies that the algebraic curve $T$ is tangent to the non-vanishing field $\mu$ and, thus, it consists of leaves of $\mathcal{G}$. A contradiction.

Thus, $G \simeq \mathbb{C}$. Since $T$ is not a leaf it must meet $G$ and furthermore $G \cap T$ is a singleton (indeed otherwise, a general integral curve of $\nu$, which is naturally isomorphic to $G \backslash T$ is hyperbolic). In fact compactness of $T$ implies that it meets every leaf of $\mathcal{G}$ at one point. Applying the flow of the nowhere-vanishing $\mu$ to $T$ we see that $Y$ is biholomorphic to $T \times \mathbb{C}$ contrary to the fact that the torus $Y$ is a compact surface. This concludes the proof of the Claim.

Thus, $Y$ is of negative Kodaira dimension. Then according to [6, Ch. 6, Prop.6] either $\mu$ is tangent to the fibers of the Albanese fibration (and, thus, has a first integral), or $Y$ can be chosen as a quadric and $\mu=\mu_{1} \oplus \mu_{2}$ where $\mu_{i}$ is a field on a factor in the direct product $Y=\mathbb{P}^{1} \times \mathbb{P}^{1}$ (and we have a natural projection $Y \rightarrow \mathbb{P}^{1}$ as an adapted Riccati fibration), or it is the suspension of a representation $\rho: \pi_{1}(\operatorname{Alb}(Y)) \rightarrow \operatorname{Aut}\left(\mathbb{P}^{1}\right)$ 
(and again the Albanese morphism $Y \rightarrow \operatorname{Alb}(Y)$ is an adapted Riccati fibration). This yields the desired conclusion.

Combining Propositions 2.9 and 2.12 with Proposition 2.3 we get the following.

Theorem 2.13. Let $\sigma$ be a complete algebraic vector field on a normal affine surface $Z$ such that $\sigma$ does not admit a rational first integral. Then there is a morphism $g: Z \rightarrow B$ onto a curve $B$ isomorphic either to $\mathbb{C}^{*}, \mathbb{C}$ or to $\mathbb{P}^{1}$ whose general fibers are isomorphic to $\mathbb{C}$ or $\mathbb{C}^{*}$ so that the flow of $\sigma$ sends fibers of $g$ to fibers of $g$.

In fact we will show in Section 5 (Theorem 5.8) that in case that the morphism $g$ is surjective to $\mathbb{P}^{1}$, there is another morphism $g_{1}: Z \rightarrow B$ with affine base $(B$ is isomorphic to $\mathbb{C}$ or $\mathbb{C}^{*}$ ) whose general fibers are isomorphic to $\mathbb{C}$ or $\mathbb{C}^{*}$ so that the flow of $\sigma$ sends fibers of $g_{1}$ to fibers of $g_{1}$.

Remark 2.14. Let us briefly discuss the alternative proof in our original preprint based on the fundamental results of foliation theory for surfaces due to Suzuki [40], [41], McQuillan [32], [31], and Brunella [8]. Suppose that we are in Setting 2.6. By a theorem of Seidenberg [39] after additional blowing-up $\hat{X} \rightarrow \bar{X}$ one can suppose that the induced foliation $\hat{\mathcal{F}}$ on $\hat{X}$ has reduced singularities only (see Definition 5.5 below).

Consider the canonical bundle $K_{\hat{\mathcal{F}}} \in \operatorname{Pic}(\hat{X}) \otimes \mathbb{Q}$ of $\hat{\mathcal{F}}$ as in Definition 2.4. One can define the Kodaira dimension of $\hat{\mathcal{F}}$ as the Kodaira-Iitaka dimension of $K_{\hat{\mathcal{F}}}$. ${ }^{7}$ In the case when there is no rational first integral for our complete field $\nu$ the crucial result of McQuillan implies that the Kodaira dimension $\operatorname{kod}(\hat{\mathcal{F}})$ is either 1 or 0 . $\operatorname{For} \operatorname{kod}(\hat{\mathcal{F}})=1$ a theorem of McQuillan yields an adapted Riccati fibration for $\hat{\mathcal{F}}$. Brunella showed that this fibration induces a $\mathbb{C}$ - or $\mathbb{C}^{*}$-fibration on $X$ whose fibers are transferred by the flow of $\nu$ into similar fibers (his proof is written for $\mathbb{C}^{2}$ but it works in general case as well).

For $\operatorname{kod}(\hat{\mathcal{F}})=0$ one has so-called McQuillan contraction $\theta: \hat{X} \rightarrow \hat{X}^{\prime}$ into a normal projective surface $\hat{X}^{\prime}$ so that $\hat{\mathcal{F}}$ induces a foliation $\hat{\mathcal{F}}^{\prime}$ on $\hat{X}^{\prime}$. Furthermore, there is a finite morphism $\rho: \hat{X}^{\prime} \rightarrow Y$ into a smooth surface $Y$ ramified at singular points of $\hat{X}^{\prime}$ only and such that it transforms $\hat{\mathcal{F}}^{\prime}$ into a foliation $\mathcal{G}$ on $Y$ generated by a global holomorphic vector field $\mu$ on $Y$. We managed to show that $\hat{X}^{\prime}$ is in fact smooth and, therefore, $\rho$ is an isomorphism. Then arguing as in Proposition 2.12 we showed that in the absence of first integrals there must be a Riccati fibration on $\mathcal{X}^{\prime}=Y$ with desired properties.

\section{Preliminaries about dual Graphs}

In this section we discuss some facts about weighted dual graphs of algebraic curves contained in smooth complete surfaces (e.g., see [11]).

Definition 3.1. Let $D$ be a closed curve contained in a smooth complete algebraic surface $\bar{X}$ such that all of its singularities are simple nodes (i.e., locally each of these

${ }^{7}$ That is,

$$
\operatorname{kod}(\hat{\mathcal{F}})=\limsup _{n \rightarrow+\infty} \frac{\log \operatorname{dim} H^{0}\left(\bar{X}, K_{\hat{\mathcal{F}}}^{\otimes n}\right)}{\log n} .
$$


singularities is a transversal intersection of two smooth analytic branches). Then we can assign the so-called weighted dual graph $\Gamma$ such that

(1) its vertices are in bijective correspondence with the irreducible components of $D$;

(2) every singularity of $D$ corresponds to an edge that joins vertices corresponding to irreducible components $C_{1}$ and $C_{2}$ that contain this singularity (if $C_{1}=C_{2}$ then this edge is a loop);

(3) each vertex is equipped with a weight that is the integer equal to the selfintersection number $C^{2}$ of the corresponding component $C$ in $\bar{X}$.

(4) We also say that $D$ is of simple normal crossing type if all components are smooth. This implies that the dual graph $\Gamma$ does not have loops.

Convention 3.2. From now on we identify the vertices of $\Gamma$ and the corresponding components of $D$ and denote them by the same letters. Furthermore, we may treat a curve contained in $D$ as a subgraph of $\Gamma$ and vice versa.

Recall that the valency of a vertex $C \in \Gamma$ is the number of edges adjacent to this vertex (with each loop counting twice) and the vertices joined with $C$ by edges are called the neighbors of $C$. The vertex is an end vertex (resp. linear vertex, resp. branch point) if its valency is 1 (resp. 2; resp. at least 3). The graph is called linear or a chain if it does not contain branch points but contains an end vertex. We use notation as $C_{1}+C_{2}+\ldots+C_{n}$ to denote such a chain with $n$ vertices in the natural order. If the weight of each $C_{i}$ is $w_{i}$ we shall also use notation $\left[\left[w_{1}, w_{2}, \ldots, w_{n}\right]\right]$ for this chain. A graph without branch points and end vertices is called circular. In this case we write $\left(\left(w_{1}, w_{2}, \ldots, w_{n}\right)\right)$ for the weights of this graph in a counterclockwise order. For any subgraph $\Gamma_{0}$ of $\Gamma$ the notation $\Gamma \ominus \Gamma_{0}$ will be used to denote the graph obtained from $\Gamma$ by removing each vertex $C \in \Gamma_{0}$ and its adjacent edges.

If $C$ is a rational irreducible component of $D$ with self-intersection $k$ we call $C$ a $k$-vertex. If $C$ is a $(-1)$-vertex with valency at most 2 in $\Gamma$ then it can be contracted and the image $D^{\prime}$ of $D$ is still a curve with nodes as singularities (unless $C$ with a loop is a connected component of $\Gamma$ - a case which we do not consider). The graph $\Gamma^{\prime}$ of $D^{\prime}$ in the smooth resulting surface can be obtained from $\Gamma \ominus C$ by joining the distinct neighbors of $C$ by an edge and increasing their weights by 1 (we call such replacement of $\Gamma$ by $\Gamma^{\prime}$ a blowing down).

A graph $\Gamma$ is contractible if it can be reduced to an empty graph by a sequence of blowing downs (i.e $D$ can be blown down to a smooth point of a resulting surface). We call $\Gamma$ minimal if it does not contain $(-1)$-vertices different from branch points.

Let $z \in D, \sigma: \hat{X} \rightarrow \bar{X}$ be the monoidal transformation of $\bar{X}$ at $z$, and $D^{\prime \prime}=\sigma^{-1}(D)$. Then the form of the dual graph $\Gamma^{\prime \prime}$ of $D^{\prime \prime}$ in $\hat{X}$ depends on whether $z$ is (a) a smooth point of $D$ (and in particular $z$ is a smooth point of an irreducible component $C$ of $D$ ) or (b) a double point of $D$, i.e., $z \in C_{1} \cap C_{2}$ where $C_{1}$ and $C_{2}$ are irreducible components of $D$. In case (a) $\Gamma^{\prime \prime}$ is obtained from $\Gamma$ by creating a new vertex of weight -1 , joining it with $C$, and reducing the weight of $C$ by 1 . This change of $\Gamma$ will be called an outer blowing up. In case (b) $\Gamma^{\prime \prime}$ is obtained from $\Gamma$ by removing the edge between $C_{1}$ and $C_{2}$, reducing their weights by 1 , and joining them by edges with a new vertex of weight -1 . Such a change of $\Gamma$ will be called an inner blowing up. 
If a graph $\Gamma_{2}$ can be obtained from a graph $\Gamma_{1}$ by a sequence of blowing up and blowing down then we call this procedure a reconstruction of $\Gamma_{1}$ into $\Gamma_{2}$. Let us give some examples of reconstructions. If $C$ is of non negative weight in a graph $\Gamma \neq C$ then making inner blowing up at an edge of $C$ one can make its weight 0 . If $C$ is a linear 0 -vertex with neighbors of weight $w_{1}$ and $w_{2}$ then making an inner blowing up at an edge of $C$ and contracting $C$ we get a reconstruction $\left[\left[w_{1}, 0, w_{2}\right]\right] \rightarrow\left[\left[w_{1}-1,0, w_{2}+1\right]\right]$. Similarly, if $C$ is end 0 -vertex with a neighbor of weight $w$ one can get $[[0, w]] \rightarrow$ $[[0, w+1]]$ or $[[0, w]] \rightarrow[[0, w-1]]$. The last three reconstructions around a 0 -vertex are called elementary transformations. The next straightforward fact will be useful.

Proposition 3.3. (see also [11, Section 2]). (1) Let $C_{1}+C_{2}+C_{3}$ be a chain with weights $w_{1}, 0, w_{2}$. Then there exist elementary transformations such that $C_{1}$ and $C_{3}$ are not blown down in this process and one has the following change of weights $\left[\left[w_{1}, 0, w_{2}\right]\right] \rightarrow$ $\left[\left[w_{1}+w_{2}, 0,0\right]\right]$.

(2) Let $C_{1}+C_{2}+\ldots+C_{n}$ be a chain (resp. a circular graph) with weights $0,0, w_{3}, \ldots, w_{n}$. Then there exist elementary transformations such that $C_{i}, \ldots, C_{n}$ are not blown down (where $3 \leq i \leq n$ ) and one has the following change of weights

$$
\begin{gathered}
{\left[\left[0,0, w_{3}, \ldots, w_{n}\right]\right] \rightarrow\left[\left[w_{3}, \ldots, w_{i}, 0,0, w_{i+1}, \ldots, w_{n}\right]\right]} \\
\left(\operatorname{resp} .\left(\left(0,0, w_{3}, \ldots, w_{n}\right)\right) \rightarrow\left(\left(w_{3}, \ldots, w_{i}, 0,0, w_{i+1}, \ldots, w_{n}\right)\right)\right) .
\end{gathered}
$$

We say that a linear graph is a standard chain if it has one the following forms: $\left[\left[0_{2 k+1}\right]\right],\left[\left[w_{1}, \ldots, w_{n}\right]\right]$, and $\left[\left[0_{2 k}, w_{1}, \ldots, w_{n-1}\right]\right]$, where $n \geq 1 k \geq 0$ and every $w_{i} \leq-2$, see (cf. [11] Definition 2.13). Here the subscript for 0 simply means the number of consecutive zeros.

We shall need later the following consequence of [11, Theorems 2.15 and Theorem 3.1 .

Proposition 3.4. Let $\Gamma_{i}, i=1,2$ be minimal non-circular graphs, $B r\left(\Gamma_{i}\right)$ be the set of branch points of $\Gamma_{i}$, and $\Gamma_{2}$ admit a reconstruction from $\Gamma_{1}$. Then

(i) $\operatorname{Br}\left(\Gamma_{i}\right)$ is an invariant of the reconstruction, i.e., none of the vertices of $\operatorname{Br}\left(\Gamma_{1}\right)$ is blown down in this procedure and they are transformed bijectively onto $\operatorname{Br}\left(\Gamma_{2}\right)$ with preservation of valency;

(ii) there is a bijection between connected components of $\Gamma_{1} \ominus \operatorname{Br}\left(\Gamma_{1}\right)$ and of $\Gamma_{2} \ominus$ $\operatorname{Br}\left(\Gamma_{2}\right)$ such that every connected component $\Gamma_{2}^{0}$ of $\Gamma_{2} \ominus \operatorname{Br}\left(\Gamma_{2}\right)$ is obtained from the corresponding connected component $\Gamma_{1}^{0}$ of $\Gamma_{1} \ominus \operatorname{Br}\left(\Gamma_{1}\right)$ by a sequence of blowing up and blowing down;

(iii) every minimal weighted graph $\Gamma_{1}$ can be reconstructed into some minimal weighted graph $\Gamma_{2}$ such that each connected component of $\Gamma_{2} \ominus \operatorname{Br}\left(\Gamma_{2}\right)$ is a standard chain;

(iv) if $\Gamma_{1}^{0}$ and $\Gamma_{2}^{0}$ are standard graphs then the reconstruction of $\Gamma_{1}^{0}$ into $\Gamma_{2}^{0}$ can be achieved by elementary transformations.

Since chains $\left[\left[w_{1}, \ldots, w_{n}\right]\right]$ with every $w_{i} \leq-2$ do not admit nontrivial elementary transformations we have the following.

Corollary 3.5. Let $\Gamma_{i}, \operatorname{Br}\left(\Gamma_{i}\right)$, and $\Gamma_{i}^{0}$ be as in Proposition 3.4. Suppose that every weight in $\Gamma_{1}^{0}$ is at most -2. Then any relatively minimal reconstruction ${ }^{8}$ of $\Gamma_{1}$ into $\Gamma_{2}$

\footnotetext{
${ }^{8}$ That is, a reconstruction without unnecessary blowing up.
} 
induces the identity transformation of $\Gamma_{1}^{0}$ into $\Gamma_{2}^{0}$. In particular, when every weight in the graph $\Gamma_{1} \ominus \operatorname{Br}\left(\Gamma_{1}\right)$ is at most -2 any relatively minimal reconstruction is the identity transformation of $\Gamma_{1}$ into $\Gamma_{2}$.

\section{4. $\mathbb{P}^{1}$-FibRATiOnS}

The results of Section 2 suggest that in order to classify complete algebraic vector fields on a semi-affine smooth surface $X$ we need to understand fibrations of $X$ with general fibers $\mathbb{C}$ or $\mathbb{C}^{*}$, for short $\mathbb{C}$ - or $\mathbb{C}^{*}$-fibrations. They can be extended to $\mathbb{P}^{1}$ fibrations (i.e., fibrations with general fiber isomorphic to $\mathbb{P}^{1}$ ) of a smooth completion $\bar{X}$ of $X$. Hence, in this section we present some general results on $\mathbb{P}^{1}$-fibrations.

Setting 4.1. For the rest of the section we suppose that $\bar{f}: \bar{X} \rightarrow B$ is a $\mathbb{P}^{1}$-fibration of a smooth projective surface over a smooth complete curve. Let $\bar{D}$ be a connected curve in $\bar{X}$ of simple normal crossing (SNC) type and $X=\bar{X} \backslash \bar{D}$ be semi-affine. We suppose that $f=\left.\bar{f}\right|_{X}$ is a $\mathbb{C}$ - or $\mathbb{C}^{*}$-fibration on $X$.

A classical result about $\mathbb{P}^{1}$-fibrations is the following, see Proposition 4.3 in [4]:

Theorem 4.2. Let $\bar{X}$ be a smooth compact surface and $C$ be a smooth rational curve in $\bar{X}$. If $C^{2}=0$, then there exists a regular birational map $\varphi: X \rightarrow Y$, where $Y$ is ruled (a $\mathbb{P}^{1}$-bundle over a curve), such that $C$ meets no exceptional curve of $\varphi$, and $\varphi(C)$ is a general fiber of $Y$.

In particular, this theorem states that singular fibers of a $\mathbb{P}^{1}$-fibration are contractible to a rational curve and, thus, their dual graphs do not contain cycles. The following lemma gives a slightly more precise statement about the singular fibers of a $\mathbb{P}^{1}$-fibration.

Lemma 4.3. In the Setting 4.1 let $\Gamma$ be the dual graph of a fiber $F=\bar{f}^{-1}(b)$ for some $b \in B$.

(1) Let $E$ be a vertex of $\Gamma$ that is reduced in $\bar{f}^{*}(b)$. Then $\Gamma \ominus E$ is contractible and, furthermore, after this contraction the weight of $E$ becomes 0.

(2) Let $E_{1}$ and $E_{2}$ be vertices of $\Gamma$ that are reduced in $\bar{f}^{*}(b)$ and let $\Gamma^{0}$ be the smallest linear subgraph of $\Gamma$ containing $E_{1}$ and $E_{2}$. Then $\Gamma \ominus \Gamma^{0}$ is contractible.

(3) Let $C$ be a vertex of $\Gamma$ that has multiplicity 2 in $\bar{f}^{*}(b)$. Then $\Gamma$ can be contracted to a graph of the form

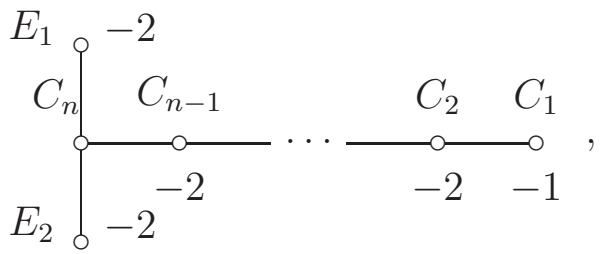

where $C_{1}$ is the proper transform of $C$ and the weight of $C_{n}$ is -2 (resp. -1$)$ for $n \geq 2$ (resp. $n=1$ ).

Proof. The first statement can be found, say, in [33, Lemma 2.11.2]. For the second statement we contract all components of $\Gamma \ominus E_{1}$ that do not contain $E_{2}$ and vice versa. By abuse of notation denote the resulting graph by the same symbol $\Gamma$. Assume that 
$\Gamma \ominus \Gamma_{0}$ is still not empty and it does not contain linear $(-1)$-vertices. It is enough to show that this is impossible.

Since any connected component of $\Gamma \ominus E_{1}$ must be contractible by (1) this assumption implies that $E_{1}$ and similarly $E_{2}$ are end-vertices of $\Gamma$. Thus, if $\Gamma \neq \Gamma^{0}$ there exists a vertex $E \in \Gamma^{0} \ominus\left(E_{1} \cup E_{2}\right)$ such that it is a branch point of $\Gamma$. Since the graph is contractible to $E_{1}$ the component of $\Gamma \ominus E$ containing $E_{2}$ must be contractible to a point in the curve $E$. This implies that $E$ is reduced in $\bar{f}^{*}(b)$ (indeed, if $E$ has multiplicity at least 2 so does $E_{2}$ because $E_{2}$ is obtained from a point in $E$ by a sequence of blowups). By (1) all components of $\Gamma \ominus E$ are contractible contradicting the assumption that $\Gamma \ominus \Gamma^{0}$ does not contain linear $(-1)$ vertices which yields $(2)$.

By Theorem 4.2 contracting consequently $(-1)$-vertices one can transform $\Gamma$ into a reduced 0 -vertex. In particular, during this contraction the proper transform of $C$ becomes a linear $(-1)$-vertex. Thus, we can suppose this is true from the beginning. Consider two cases: (i) $C$ is not an end vertex of $\Gamma$ and (ii) $C$ is an end vertex of $\Gamma$. Let $E_{1}$ and $E_{2}$ be the neighbors of $C$ in (i). Note that $E_{1}$ and $E_{2}$ are reduced in $\bar{f}^{*}(b)$ since the multiplicity of $C$ in $\bar{f}^{*}(b)$ is the sum of the corresponding multiplicities of $E_{1}$ and $E_{2}$. Hence, by (2) $\Gamma$ can be contracted to the chain $E_{1}+C+E_{2}$ and its weights must be $-2,-1,-2$ since otherwise $\Gamma$ cannot be contracted to a 0 -vertex. In (ii) contracting $C$ we observe that its neighbor must have multiplicity 2 in $\bar{f}^{*}(b)$. Thus, replacing $C$ by this neighbor and using the induction by the number of vertices we conclude that $\Gamma$ can be contracted to a graph of the form presented in statement (3) which concludes the proof.

Definition 4.4. If $f: X \rightarrow B$ is a $\mathbb{C}^{*}$-fibration then $\bar{D}$ either contains two sections $B_{1}$ and $B_{2}$ of $\bar{f}$ (we call this case untwisted) or it contains a curve $B_{0}$ such that $\left.\bar{f}\right|_{B_{0}}: B_{0} \rightarrow B$ is a ramified double cover of rational curves (so-called twisted case).

Remark 4.5. It is worth mentioning that in the twisted case a fiber $\bar{f}^{-1}(b), b \in B$ meets $B_{0}$ at one point if and only if $b$ is a ramification point of the morphism $\left.\bar{f}\right|_{B_{0}}: B_{0} \rightarrow B$. The number of ramifications points is determined by the Riemann-Hurwitz formula and when $B_{0} \simeq \mathbb{P}^{1}$ there are exactly two of them.

Proposition 4.6. In the Setting 4.1 let $F=\bar{f}^{-1}(b)$ be a fiber contained in $\bar{D}$. Suppose that the dual graph $\Gamma$ of $\bar{D}$ does not contain linear $(-1)$-vertices different from irreducible curves on which the restriction of $\bar{f}$ is non-constant (we call such $\bar{D}$ pseudominimal). Let $\Gamma_{0}$ be the smallest subgraph of $\Gamma$ that contains all components of $F$ and their neighbors.

(1) If $f$ is a $\mathbb{C}$-fibration then $F$ is a 0 -vertex and $\Gamma_{0}$ is a linear chain $F+B^{\prime}$ where $B^{\prime}$ is a section of $\bar{f}$.

(2) If $f$ is an untwisted $\mathbb{C}^{*}$-fibration then $F$ is a 0 -vertex and $\Gamma_{0}$ is a linear chain $B_{1}+F+B_{2}$.

(3) Let $f$ be a twisted $\mathbb{C}^{*}$-fibration.

(3a) Suppose that $F$ meets $B_{0}$ at two points. Then $F$ is a 0 -vertex and $\Gamma_{0}$ is a cycle consisting of $F$, and $B_{0}$ joined by two edges. 
(3b) Suppose that $F$ meets $B_{0}$ at one point. Then $F$ is a linear chain $C_{1}+E+C_{2}$ where $C_{1}$ and $C_{2}$ are (-2)-vertices, $E$ is a (-1)-vertex, and $\Gamma_{0} \ominus E$ contains three components $C_{1}, C_{2}$, and $B_{0}$. That is, the following

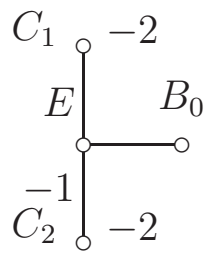

is the form of $\Gamma_{0}$ (in the rest of the paper subgraphs of $\Gamma$ satisfying the assumptions of (3b) will be called subgraphs of type $\Gamma_{*}$ ).

Proof. Note that in (1) $B^{\prime}$ is the only neighbor of $F$ in $\Gamma$ since otherwise $f$ is not a $\mathbb{C}$-fibration. The component $E$ of $F$ that meets $B^{\prime}$ is reduced in $\bar{f}^{*}(b)$ because $f^{*}(b) \cdot B^{\prime}=1$. By Lemma 4.3 one can contract $F$ to $E$ but since $F$ does not have linear $(-1)$-vertices we have $F=E$.

Since $f$ is a $\mathbb{C}^{*}$-fibration in (2), by similar reasons $B_{1}$ and $B_{2}$ are the only neighbors of $F$ in $\Gamma$ and the component $E_{i}$ of $F$ that meets $B_{i}$ is reduced in $\bar{f}^{*}(b)$. By Lemma 4.3 and pseudo-minimality $F$ must be a linear chain with $E_{1}$ and $E_{2}$ being end vertices. In particular, $E_{1}$ and $E_{2}$ are linear vertices of $\Gamma$. Note that if $E_{1} \neq E_{2}$ then both of them are $(-1)$-curves since the curve $\overline{F \backslash E_{i}}$ is contractible by Lemma 4.3. This contradicts pseudo-minimality. Thus, $E_{1}=E_{2}=F$ which yields (2).

In (3a) as before we have $B_{0}$ as the only neighbor of $F$ in $\Gamma$ and the neighbors of $B_{0}$ in $F$ are $E_{1}$ and $E_{2}$ which are reduced in $\bar{f}^{*}(b)$ (indeed we have $f^{*}(b) \cdot B_{0}=2$ ). Again, by Lemma 4.3 and pseudo-minimality we see that $F=E_{1}=E_{2}$, i.e., being an irreducible fiber of $\mathbb{P}^{1}$-fibration $F$ is the 0 -vertex which is (3a).

In (3b) $B_{0}$ is again the only neighbor of $F$ in $\Gamma$. More precisely $B_{0}$ is a neighbor of a vertex $E$ in the graph $\Gamma_{1}$ of $F$ and $E$ has multiplicity 2 in $\bar{f}^{*}(b)$ since for the double section $B_{0}$ one has $\bar{f}^{*}(b) \cdot F=2$. Since $\Gamma_{1}$ is contractible to a 0 -vertex it contains a linear $(-1)$-vertex. The assumption on $(-1)$-vertices implies that this vertex is not in $\Gamma_{1} \ominus E$. Hence, $E$ is a linear $(-1)$-vertex in $\Gamma_{1}$. Let $C_{1}$ and $C_{2}$ be its neighbors in $\Gamma_{1}$. Note that they are reduced in $\bar{f}^{*}(b)$. Indeed, since $E$ is contractible to the points of intersection of the images of $C_{1}$ and $C_{2}$ the multiplicity of $C$ is the sum of multiplicities of $C_{1}$ and $C_{2}$, i.e., $2=1+1$. By Lemma 4.3 one can contract all components of $\Gamma_{1} \ominus\left(C_{1} \cup E \cup C_{2}\right)$ and we are done.

Remark 4.7. Let $C$ be a smooth rational curve in $\bar{X}$ with $C^{2}=0$ (i.e., it is a fiber of a $\mathbb{P}^{1}$-fibration by Theorem 4.2). The following converse of Proposition 4.6 is true.

(1) Let $C$ be an end vertex of $\Gamma$, then $X$ admits a $\mathbb{C}$-fibration $f$ such that $C$ is a fiber of $\bar{f}$.

(2) Let $C$ be a linear vertex of $\Gamma$ with two distinct neighbors $B_{1}$ and $B_{2}$. Then $X$ admits an untwisted $\mathbb{C}^{*}$-fibration $f$ such that $C$ is a fiber of $\bar{f}$ and $B_{1}$ and $B_{2}$ are sections of $\bar{f}$. 
(3a) Let $C$ be a linear vertex of $\Gamma$ with one neighbor $B_{0}$ only (i.e., $C$ is joined with $B_{0}$ by two edges). Then $X$ admits a twisted $\mathbb{C}^{*}$-fibration $f$ such that $C$ is a fiber of $\bar{f}$ and $B_{0}$ is the double section $\bar{f}$ which intersects $C$ transversally in two points.

(3b) Let $C_{1}+C+C_{2}$ be a linear subgraph of $\Gamma$ as in (3b) with $E$ replaced by $C$ and $B_{0}$ being the only neighbor of $C$ different from $C_{1}$ and $C_{2}$. Then $X$ admits a twisted $\mathbb{C}^{*}$-fibration $f$ such that $C_{1} \cup C \cup C_{2}$ is a singular fiber of $\bar{f}$ and $B_{0} \subset \bar{D}$ is the double section of $\bar{f}$.

We need one more technical fact for Section 10.

Lemma 4.8. In the Setting 4.1 let $\bar{f}: \bar{X} \rightarrow B$ be a pseudo-minimal extension of $f$, and $U$ be the union of components of $\bar{D}$ on which the restriction of $\bar{f}$ is not constant. Suppose that $E_{1}$ and $E_{2}$ are the only irreducible components of $\bar{f}^{-1}(b), b \in B$ that meet the divisor $U$ (where we allow equality $E_{1}=E_{2}$ ) and that $\Gamma^{0}$ is as in Lemma 4.3.

(i) Let $F$ be an irreducible affine $e^{9}$ component of $f^{-1}(b)$ whose closure is not a vertex in $\Gamma^{0}$. Then $F \simeq \mathbb{C}$.

(ii) If the dual graph $\Gamma^{\prime}$ of $\bar{f}^{-1}(b) \cap \bar{D}$ is not contractible then $f^{-1}(b)$ is a singular fiber of the fibration $f$ (in other words, for every neighborhood $W$ of $b$ in $B$ the map $\left.f\right|_{f^{-1}(W)}: f^{-1}(W) \rightarrow W$ is not a locally trivial fibration even in the sense of differential topology).

Proof. The dual graph $\Gamma^{\prime \prime}$ of the fiber $f^{-1}(b)$ is a tree since otherwise $\Gamma^{\prime \prime}$ cannot be contracted to a 0 -vertex. Let $\Gamma^{1}$ be the subgraph of $\Gamma^{\prime \prime} \backslash \Gamma^{0}$ consisting of the vertices corresponding to the irreducible components of $\bar{f}^{-1}(b)$ meeting $f^{-1}(b)$. Since $X$ is semi-affine and, thus, by the Lefschetz hyperplane section theorem $\bar{D}$ is connected a vertex $\bar{F} \in \Gamma^{1}$ can be only an endpoint of $\Gamma^{\prime \prime} \ominus \Gamma^{0}$ which is not a neighbor of $\Gamma^{0}$. This implies that $\bar{F}$ is an endpoint in $\Gamma^{\prime \prime}$ which yields (i).

If $f^{-1}(b)$ is not singular then this fiber is reduced irreducible. Its closure is can be viewed as a vertex $V$ in $\Gamma^{\prime \prime}$ such that $\Gamma^{\prime}=\Gamma^{\prime \prime} \ominus V$. By Lemma $4.3 \Gamma^{\prime}$ is contractible which is the desired conclusion.

\section{ON NON-AFFINE BASE OF RICCATI FIBRATION}

The aim of this section is to show that under the assumptions of Theorem 2.13 with $B \simeq \mathbb{P}^{1}$, there is another Riccati fibration for the same vector field which is not surjective. The first step will be to prove that the $Z$ is a toric surface. The existence of a Riccati fibration with affine base in this case has been established in [27] (heavily using Brunella's result for $\mathbb{C}^{2}$ ).

Let us prove first several technical facts.

Lemma 5.1. Let $X$ be a normal compact complex surface, $M$ be a finite subset of $X$, and $X^{*}=X \backslash M$. Suppose that $\nu$ is a complete holomorphic vector field on $X^{*}$. Then $\nu$ extends to a similar field on $X$.

Proof. Let $\varphi_{t}: X \rightarrow X, t \in \mathbb{C}$ be the flow of $\nu$ and $x \in M$. When $t$ has a small absolute value then by continuity $\varphi_{t}$ transforms a punctured Stein neighborhood $U_{x}$ of

\footnotetext{
${ }^{9}$ That is, a component that survives the Remmert reduction of $X$.
} 
$x$ into a similar Stein neighborhood $U_{x}^{\prime}$. Thus, by Hartogs' theorem $\varphi_{t}$ extends to $x$ which implies the desired conclusion.

Lemma 5.2. Let the curve $B=g(Z)$ in Theorem 2.13 be isomorphic to $\mathbb{P}^{1}$ and let $\pi: X \rightarrow Z$ be a minimal resolution of singularities of $Z$. Then there exists a smooth $S N C$-completion $\bar{X}$ of $X$ so that the lift of $\sigma$ to $X$ extends to an algebraic vector field $\bar{\sigma}$ on $\bar{X}$.

Proof. By the assumption of Theorem 2.13 there exists a complete nontrivial algebraic vector field $\nu$ on $B$ for which $f_{*}(\sigma)=\nu$. Let $O$ be the open orbit of $\nu$ in $B$ (i.e., either $O \simeq \mathbb{C}$ or $\left.O \simeq \mathbb{C}^{*}\right)$ and let $U=f^{-1}(O)$. If $F=g^{-1}(z)$ for a general point $z \in O$ (i.e., either $F \simeq \mathbb{C}$ or $F \simeq \mathbb{C}^{*}$ ) then using the flow of $\sigma$ one can see that $U$ is biholomorphic to $F \times O$ over $O$. In particular, all fibers of the morphism $\left.g\right|_{U}$ are reduced and isomorphic to $F$. Let us extend $\left.g\right|_{U}$ to a proper morphism $\bar{g}: \bar{U} \rightarrow O$ from a smooth surface $\bar{U}$ which is, of course, a $\mathbb{P}^{1}$-fibration. Since the closure of $F$ in $\bar{U}$ is a reduced component of the fiber $\bar{g}^{-1}(z)$ we can contract all other components of this fiber by Lemma 4.3 (1). That is, $\bar{U}$ is naturally isomorphic to $\mathbb{P}^{1} \times O$.

Furthermore, one can see that because of completeness the field $\sigma$ is tangent to $\bar{U} \backslash U$, i.e., it can be extended to a complete algebraic field on $\bar{U}$. Thus, we have an algebraic extension of $\sigma$ to the surface $Z^{\prime}=Z \cup \bar{U}$. Since this extension vanishes at the singular points we can blow these points up and obtain a complete algebraic vector field $\delta$ on the smooth surface $X^{\prime}=X \cup \bar{U}$ that extends $\left.\sigma\right|_{\bar{U}}$. Extend the natural projection $g^{\prime}: X^{\prime} \rightarrow B$ further to a proper morphism $\bar{g}^{\prime}: \bar{X} \rightarrow B$ such that it coincides with $\bar{g}$ over $O$. Note that the curve $\left(\bar{g}^{\prime}\right)^{-1}(B \backslash O) \backslash X^{\prime}$ has a negative definite intersection form by the Zariski lemma [4, page 90]. Thus, by the Grauert criterion [4] it can be contracted. The result of this contraction $\rho: \bar{X} \rightarrow \hat{X}$ is a normal complex surface $\hat{X}$ such that it contains $X^{\prime}$ and $\hat{X} \backslash X^{\prime}$ is finite. By Lemma $5.1 \delta$ extends to a complete holomorphic vector field on $\hat{X}$. Blowing up the singular points of $\hat{X}$ we can lift this complete holomorphic vector field to a complete holomorphic vector field $\bar{\sigma}$ on $\bar{X}$. Since $\bar{X}$ is a complete algebraic surface this field $\bar{\sigma}$ is algebraic [4] which concludes the proof.

Remark 5.3. The assumption that $B$ is a complete curve is crucial for Lemma 5.2. In its absence one can construct a complete vector field on a smooth affine surface $Z$ that do not admit a regular extension to any completion $\bar{Z}$ of $Z$. Indeed, for $Z=\mathbb{C}^{*} \times \mathbb{C}$ with a coordinate $x$ and $z$ on the first and the second factors respectively consider a vector field of the form $\nu=x \frac{\partial}{\partial x}-x^{-k}(P(x) z+Q(x)) \frac{\partial}{\partial z}$ where $k \geq 0$ and $P$ and $Q$ are polynomials. In this case $B=\mathbb{C}^{*}$ and $g: Z \rightarrow B$ is the natural projection. We can suppose that $g$ extends to $\bar{g}: \bar{Z} \rightarrow \mathbb{P}^{1}$. However, it is not difficult to see that the extension of $\nu$ has poles either on $\bar{g}^{-1}(0)$ or on $\bar{g}^{-1}(\infty)$.

Lemma 5.4. (1) Let $\bar{g}: \bar{X} \rightarrow B, X$ and $\bar{\sigma}$ be as in Lemma 5.2 (in particular, $\bar{\sigma}$ does not have a rational first integral and $\bar{g}$ is a surjective map onto $B \simeq \mathbb{P}^{1}$ ). Suppose that $Y=\mathbb{P}^{1} \times \mathbb{P}^{1}$ where the first (resp. second) factor is equipped with an affine coordinate $z$ (resp. w). Then there exists a non-regular birational map $\chi: \bar{X}-\rightarrow Y$ such that it transforms $\bar{\sigma}$ into a complete holomorphic vector field $\mu=\mu_{1}+\mu_{2}$ on $Y$ where either

(a) $\mu_{1}=\alpha z \frac{\partial}{\partial z}$ and $\mu_{2}=\beta w \frac{\partial}{\partial w}$ with $\alpha$ and $\beta \in \mathbb{C}$ being linearly independent over $\mathbb{Q}$, or 
(b) $\mu_{1}=\frac{\partial}{\partial z}$ and $\mu_{2}=\gamma w \frac{\partial}{\partial w}$ with $\gamma \neq 0$.

(2) Furthermore, let $C_{1}=\{w=0\}, C_{2}=\{z=\infty\}, C_{3}=\{w=\infty\}$, and $C_{4}=\{z=$ $0\}$ be the lines in $Y$. Let $R=\bigcup_{i=1}^{4} C_{i}$ in case (a) and $R=\bigcup_{i=1}^{3} C_{i}$ in case (b). Then the restriction of $\chi$ over $Y \backslash R$ is an isomorphism and $\chi^{-1}: Y \rightarrow \bar{X}$ is regular outside the set $P$ of singular points of $R$.

(3) Suppose that $D=\bar{X} \backslash X$ and $T=D \cup \bar{g}^{-1}(B \backslash O)$ (where $O$ is as in the proof of Lemma 5.2). Then $\chi(T) \subset R$.

Proof. By [6, Section 6, Theorem 6 (iii)] there exists $\chi: \bar{X}-\rightarrow Y$ such that it transforms $\bar{\sigma}$ into a complete holomorphic vector field $\mu=\mu_{1}+\mu_{2}$ on $Y=\mathbb{P}^{1} \times \mathbb{P}^{1}$ where $\mu_{i}$ is the lift of a complete vector field on the $i$-th factor of $Y$. This implies that, say, $\mu_{1}$ is of the form $l(z) \frac{\partial}{\partial z}$ where $l(z)$ is a quotient of linear polynomials. However, some of these fields $\mu$ have rational first integrals (e.g., $\mu=\alpha \frac{\partial}{\partial z}+\beta \frac{\partial}{\partial w}$ with $\alpha$ and $\beta \in \mathbb{C}$ has the rational first integral $\beta z-\alpha w)$. Disregarding such fields we see that up to automorphisms of the factors $\mathbb{P}^{1}$ we are left with the choices presented in cases (a) and (b) which is (1).

It follows from the proof of $[6$, Section 6 , Theorem 6 (iii)] that $\chi$ is a composition of a sequence of monoidal transformations and contractions of $(-1)$-curves where each monoidal transformation occurs at a point where the vector field (induced by) $\bar{\sigma}$ is zero while for every contracted (-1)-curve such a field either is tangent to it or vanishes on it (in particular, the image of this curve after the contraction is a point where the consequent induced vector field is zero).

Note that $\mu$ does not vanish on $Y \backslash R$ and none of integral curves of $\mu$ contained in $Y \backslash R$ is algebraic, i.e., such curves cannot be contracted under $\chi^{-1}: Y \rightarrow \bar{X}$. Thus, $\chi$ is an isomorphism over $Y \backslash R$. Furthermore, $\mu$ vanishes only at $P$ and, therefore, the indeterminacy points of $\chi^{-1}$ are contained in $P$ which concludes the proof of (2).

By construction $T$ consists of irreducible components invariant under the foliation induced by $\bar{\sigma}$. Hence, $\chi(T)$ consists of irreducible components invariant under the foliation induced by $\mu$. Furthermore, $\chi(T)$ is an algebraic curve since $D$ is. Since all algebraic curves tangent to $\mu$ are contained in $R$ we get (3).

Definition 5.5. Let $\nu$ be a holomorphic vector field on a smooth complex surface $X$ vanishing at a point $x_{0} \in X$. Then $x_{0}$ is a reduced singularity of $\nu$ if the linear part of $\nu$ at $x_{0}$ has eigenvalues $\lambda_{1}, \lambda_{2}$ such that either they are nonzero and $\lambda_{1} / \lambda_{2} \notin \mathbb{Q}$ or $\lambda_{1} \neq 0=\lambda_{2}$ (e.g., every point $p \in P$ from Lemma 5.4 is a reduced singularity for the vector fields $\mu$ described in that lemma).

The main property of reduced singularities we are going to exploit is the following. Let $\pi: \tilde{X} \rightarrow X$ be the blowing up of $X$ at $x_{0}$ and $E \simeq \mathbb{P}^{1}$ be its exceptional divisor. Then $\nu$ induces the vector field $\tilde{\nu}$ on $\tilde{X}$ such that it is tangent to $E$, vanishes exactly at two points of $E$, and has both of these singularities reduced. ${ }^{10}$

Lemma 5.6. Let the assumption of Lemma 5.4 hold. Then there exist a smooth surface $W$ and morphisms $\varphi: W \rightarrow \bar{X}$ and $\psi: W \rightarrow Y$ such that $\chi=\psi \circ \varphi^{-1}$ and the dual

\footnotetext{
${ }^{10}$ One can extract this from the following computation. Suppose that $\alpha z \frac{\partial}{\partial z}+\beta w \frac{\partial}{\partial w}$ is the linear part of $\nu$ in a local coordinate system $(z, w)$ at $x_{0}$. Then we can consider a local system $(\xi, \eta)$ on $\tilde{X}$ such that $(z, w)=(\xi, \xi \eta)$. The restriction of $\tilde{\nu}$ to $E$ is given by $\alpha \xi \frac{\partial}{\partial \xi}+(\beta-\alpha) \eta \frac{\partial}{\partial \eta}$.
} 
graph of the curve $\psi^{-1}(R)$ is either circular or linear. Furthermore, the vector fields $z \frac{\partial}{\partial z}$ and $w \frac{\partial}{\partial w}$ on $Y$ induce holomorphic vector field $\delta_{1}$ and $\delta_{2}$ on $W$ such that they are tangent to every component of $\psi^{-1}(R)$.

Proof. The existence of $\varphi: W \rightarrow \bar{X}$ and $\psi: W \rightarrow Y$ such that $\chi=\psi \circ \varphi^{-1}$ follows from the Zariski theorem [4]. We suppose also that these morphisms are chosen so that there are no curves in $W$ that are contracted by both $\varphi$ and $\psi$. Then according to the proof of $[6$, Section 6, Theorem 6 (iii)] $\psi$ is a composition of a sequence of monoidal transformations each of which occurs at a point where the vector field (induced by) $\mu$ vanishes. Since $\mu$ vanishes at $P$ only the first of these transformations $Y^{\prime} \rightarrow Y$ takes place at a point $p \in P$. It transforms $R$ to a curve $R^{\prime}$. Since the dual graph $\Gamma$ of $R$ is linear or circular and $p$ is a double point of $R$ the dual graph $\Gamma^{\prime}$ of $R^{\prime}$ is obtained from $\Gamma$ by an inner transformation (see Section 3 for the definition of an inner transformation) and, therefore, $\Gamma^{\prime}$ remains linear or circular. Let $\mu^{\prime}$ (resp. $\delta_{1}^{\prime}$, resp. $\delta_{2}^{\prime}$ ) be the vector field on $Y^{\prime}$ induced by $\mu$ (resp. $z \frac{\partial}{\partial z}$, resp. $w \frac{\partial}{\partial w}$ ) and $E \subset R^{\prime}$ be the exceptional curve of the transformation. As we mentioned before there are only two points on $E$ where $\mu^{\prime}$ vanishes (and they are reduced singularities of $\mu^{\prime}$ ). The same is true for $\delta_{1}^{\prime}$ and $\delta_{2}^{\prime}$. Since the whole curve $R^{\prime}$ is invariant under the flow of $\mu^{\prime}$ (resp. $\delta_{1}^{\prime}$, resp. $\delta_{2}^{\prime}$ ) these two points must be automatically the points where $E$ meets other irreducible components of $R^{\prime}$. That is, they are again double points of the simple normal crossing curve $R^{\prime}$. As we mentioned before the consequent monoidal transformation must occur at a zero of $\mu^{\prime}$ (and such a zero is simultaneously a zero of $\delta_{1}^{\prime}$ and of $\delta_{2}^{\prime}$ ). This leads to an inner transformation of $\Gamma^{\prime}$. In particular, the absence of branch points for the dual graphs is preserved on this step and similarly on all consequent steps while the lifts of $z \frac{\partial}{\partial z}$ and $w \frac{\partial}{\partial w}$ remain holomorphic and tangent to the preimage of $R$. This yields the desired conclusion.

Proposition 5.7. Let the assumptions of Theorem 2.13 hold and the fibration $g$ being onto $B \simeq \mathbb{P}^{1}$. Then $Z$ is a toric surface.

Proof. By Lemma 5.4 we have $D^{\prime}=\psi^{-1}(D)$ and $T^{\prime}=\psi^{-1}(T)$ contained in $R^{\prime}=$ $\psi^{-1}(R)$. By Lemma 5.6 the dual graph of $R^{\prime}$ is either circular or linear. If $D^{\prime}=R^{\prime}$ then $T^{\prime} \backslash D^{\prime}=\varnothing$, i.e., $\bar{g}$ has no fibers invariant under the foliation induced by $\bar{\sigma}$. Hence, $\bar{g}$ is not surjective contrary to the assumption. Thus, the dual graph of $D^{\prime}$ is linear. Therefore, the dual graph of $D$ is linear which implies already that $Z$ is a Gizatullin surface [16]. Furthermore, by Lemma 5.4 the restriction of $\chi$ over $Y \backslash R$ is an isomorphism which implies that the curves contracted by the birational morphism $\varphi: W \rightarrow \bar{X}$ are contained in $R^{\prime}$. Since these curves are tangent to the vector fields $\delta_{1}$ and $\delta_{2}$ on $W$ (from Lemma 5.6) we can push these fields down to holomorphic vector fields on $\bar{X}$ tangent to $D$. That is, these resulting fields are induced (via $\chi$ ) by the vector fields $z \frac{\partial}{\partial z}$ and $w \frac{\partial}{\partial w}$ on $Y$ whose flows commute and generate the action of the torus $\left(\mathbb{C}^{*}\right)^{2}$ on $Y$. This implies that we have the torus action on $\bar{X}$ and $X$. Every complete curve in $X$ has a negative self-intersection and, thus, it is invariant under the action. Contraction of such curves leads to a non-degenerate torus action on $Z$ which concludes the proof.

Theorem 5.8. Let $\sigma$ be a complete algebraic vector field on a normal affine surface $Z$ such that $\sigma$ does not admit a rational first integral. Then there is a regular function 
$g: Z \rightarrow \mathbb{C}$ whose general fibers are isomorphic to $\mathbb{C}$ or $\mathbb{C}^{*}$ so that the flow of $\sigma$ sends fibers of $g$ to fibers of $g$.

Proof. We have to consider the case when the fibration provided by Theorem 2.13 is surjective onto $B \simeq \mathbb{P}^{1}$. By Proposition 5.7 this can only happen when we are dealing with a toric surface $Z$. For toric surfaces the existence of another Riccati fibration with affine base has been deduced in [27] (Lemma 4.3) from Brunella's original work.

\section{RATIONAL FIRST INTEGRAL}

The aim of this section is to classify generalized Gizatullin surfaces admitting nonzero complete algebraic vector fields that have non-regular first integrals.

Proposition 6.1. Let $B$ be a germ of a smooth curve at point $o$ and let $\mu$ be the vector field on $B \times \mathbb{P}_{x}^{1}$ given by $\mu=x \partial / \partial x$ where $\mathbb{P}_{x}^{1}=\mathbb{C} \cup \infty$ and $\mathbb{C}$ is equipped with a coordinate $x$ (i.e., the set of its zeros of $\mu$ is the union of $B \times\{\infty\}$ and $B \times\{0\}$ ). Suppose that pr: $\tilde{X} \rightarrow B$ is a smooth $\mathbb{P}^{1}$-fibration and $\psi: \tilde{X} \rightarrow B \times \mathbb{P}_{x}^{1}$ is a birational morphism over $B$ whose restriction over $B \backslash$ o is an isomorphism. Let the dual graph of $\mathrm{pr}^{-1}(o)$ be linear with end vertices meeting the proper transforms $\tilde{B}_{1}$ and $\tilde{B}_{2}$ of $B \times\{\infty\}$ and $B \times\{0\}$ respectively. Then $\mu$ induces a regular complete vector field $\tilde{\mu}$ on $\tilde{X}$ tangent to the fibers of $\mathrm{pr}$ and such that its restriction to $\mathrm{pr}^{-1}(o)$ vanishes only at double points of the curve $\tilde{B}_{1} \cup \operatorname{pr}^{-1}(o) \cup \tilde{B}_{2}$.

Proof. We use induction on the number $k$ of irreducible components of $\operatorname{pr}^{-1}(o)$. If $k=1$ then $\psi$ is an isomorphism and there is nothing to prove. Note that contracting a $(-1)$-curve in $\operatorname{pr}^{-1}(o)$ we obtain a birational morphism $\sigma: \tilde{X} \rightarrow \breve{X}$ over $B$ such that $\psi$ factors through it and the dual graph of $\mathrm{pr}^{-1}(o)$ (for the natural projection $\breve{p r}: \breve{X} \rightarrow B)$ is linear with end vertices meeting the proper transforms $\breve{B}_{1}$ and $\breve{B}_{2}$ of $B \times\{\infty\}$ and $B \times\{0\}$ respectively. By assumption $\mu$ induces a regular complete vector field $\breve{\mu}$ on $\breve{X}$ tangent to the fiber of $\breve{p r}$ and such that its restriction to $\mathrm{pr}^{-1}(o)$ vanishes only at double points of the curve $\breve{B}_{1} \cup \breve{p r}^{-1}(o) \cup \breve{B}_{2}$.

Note that $\sigma$ is a monoidal transformation with center also at one of these points. Hence, $\breve{\mu}$ generates a field $\tilde{\mu}$ tangent to the fibers of pr which is complete by construction. Its flow preserves the curve $\tilde{B}_{1} \cup \mathrm{pr}^{-1}(o) \cup \tilde{B}_{2}$ and, in particular, it keeps the set $S$ of the double points of this curve fixed, i.e $\tilde{\mu}$ vanishes at these points. For any component $F$ of $\operatorname{pr}^{-1}(o)$ on which $\tilde{\mu}$ is not identically zero, $\tilde{\mu}$ does not vanish on $F \backslash S \simeq \mathbb{C}^{*}$ since no rational curve but $\mathbb{C}$ or $\mathbb{C}^{*}$ can be an integral curve of a complete vector field. Therefore, it remains to show that $\tilde{\mu}$ has only isolated zeros on $\operatorname{pr}^{-1}(o)$.

By induction one can suppose that in some local coordinate system $(z, w)$ near a double point of $\breve{B}_{1} \cup \breve{p r}^{-1}(o) \cup \breve{B}_{2}$ the local equation of $\breve{B}_{1} \cup \breve{p r}^{-1}(o) \cup \breve{B}_{2}$ is $z w=0$ and the field $\breve{\mu}$ coincides with

$$
n z \frac{\partial}{\partial z}-m w \frac{\partial}{\partial w}
$$

for natural $n$ and $m$ with $n+m \geq 1$. Furthermore, for a local coordinate system $(\xi, \eta)$ on $\tilde{X}$ the map $\sigma$ is given by $(z, w)=(\xi, \xi \eta)$. The direct computation shows that the local form of $\tilde{\mu}$ is

$$
n \xi \frac{\partial}{\partial \xi}-(n+m) \eta \frac{\partial}{\partial \eta}
$$


which implies the desired conclusion.

Remark 6.2. Note that $\mu$ is semi-simple, i.e., its flow is an algebraic $\mathbb{C}^{*}$-action on $B \times \mathbb{P}^{1}$. Hence, the flow of $\tilde{\mu}$ is also an algebraic $\mathbb{C}^{*}$-action on $\tilde{X}$ and $\tilde{\mu}$ is semi-simple as well.

Setting 6.3. Let $\nu$ be a complete vector field on a smooth semi-affine surface $X$ with a rational first integral. Blowing up $X$ at the points of indeterminacy (note that $\nu$ vanishes at such points) we can suppose that this integral is a regular morphism $f: X \rightarrow B$ where $B$ is a complete curve. In particular, $f$ is either a $\mathbb{C}$ - or a $\mathbb{C}^{*}$-fibration. Let $\hat{f}: \hat{X} \rightarrow B$ be an extension of $f$ to a $\mathbb{P}^{1}$-fibration on a smooth completion $\hat{X}$ of $X$ by an SNC-divisor $\hat{D}$ which is assumed to be pseudo-minimal. Similarly, we suppose that the union $\hat{E}$ of complete curves in $\hat{X}$ does not contain $(-1)$-curves tangent to $\nu$ (and we call such $\hat{E}$ pseudo-minimal). The extension of $\nu$ to $\hat{X}$ will be denoted by $\hat{\nu}$ (i.e., $\hat{\nu}$ may have poles). We note that unless $f$ is a twisted $\mathbb{C}^{*}$-fibration the set of zeros of $\hat{\nu}$ contains

(a) either only one section $B_{0}$ of $\hat{f}$ (i.e a general integral curve of $\hat{\nu}$ is isomorphic to $\mathbb{C}$ ) or

(b) two sections $B_{1}$ and $B_{2}$ of $\hat{f}$ (i.e a general integral curve of $\hat{\nu}$ is isomorphic to $\mathbb{C}^{*}$ )

where the second option is automatic for the untwisted $\mathbb{C}^{*}$-fibration. Furthermore, $B_{0}$ (resp. at least $B_{1}$ ) must be contained in $\hat{D}$ since otherwise $X$ is not semi-affine.

Lemma 6.4. In the Setting 6.3 let either (a) or (b) hold. Suppose that $\mu=\partial / \partial x$ (resp. $\mu=x \partial / \partial x)$ is the vector field on $B \times \mathbb{P}_{x}^{1}$ in case (a) (resp. (b)). Then there exists a birational map $\varphi: \hat{X} \rightarrow B \times \mathbb{P}^{1}$ over $B$ (in particular the restriction of $\varphi$ over some open Zariski dense subset $B^{*}$ of $B$ is an isomorphism) for which

(1) $\mu$ induces a rational vector field $\hat{\mu}$ on $\hat{X}$ such that for some rational function $p$ on $B$ one has $\hat{\nu}=\hat{f}^{*}(p) \hat{\mu}$;

(2) in case (b) the restriction of $\hat{\mu}$ to any fiber of $\hat{f}$ has a finite number of zeros;

(3) if in case (b) $\left.\hat{\mu}\right|_{X}$ is regular then $\hat{\mu}$ is semi-simple and the dual graph of the curve $B_{1} \cup \hat{f}^{-1}(b) \cup B_{2}$ is linear for every $b \in f(X)$.

Proof. By Theorem 4.2 there is a birational morphism $\tau: \hat{X} \rightarrow Y$ onto a ruled surface $Y$ over $B$. Recall that $Y$ is a locally trivial $\mathbb{P}^{1}$-fibration over $B$ [22, Prop. V.2.2], i.e., there is a Zariski dense open subset $B^{*}$ of $B$ such that the preimage $Y^{*}$ of $B^{*}$ under the projection $Y \rightarrow B$ is naturally isomorphic to $B^{*} \times \mathbb{P}^{1}$ (more precisely, such $B^{*}$ can be chosen as a neighborhood of any $b_{0}$ for which the fiber $f^{-1}\left(b_{0}\right)$ is not a singular one). Furthermore, reducing $B^{*}$ one can suppose that the proper transforms of $B_{1}$ and $B_{2}$ in $Y^{*}$ (in case (b)) are disjoint and the restriction of $\tau$ to $\hat{X}^{*}=\tau^{-1}\left(Y^{*}\right)$ yields an isomorphism $X^{*} \simeq Y^{*}$. Using the freedom of choice of this isomorphism $Y^{*} \simeq B^{*} \times \mathbb{P}^{1}$ one can suppose now that these proper transforms are $B^{*} \times\{\infty\}$ and $B^{*} \times\{0\}$ respectively (and in case (a) the proper transform of $B_{0}$ is $B^{*} \times\{\infty\}$ ). Extending the isomorphism $Y^{*} \simeq B^{*} \times \mathbb{P}^{1}$ we get $\varphi: \hat{X} \rightarrow B \times \mathbb{P}^{1}$. Let us show that $\varphi$ is the desired rational map.

By the Zariski theorem there is a surface $W$ that dominates both $\hat{X}$ and $B \times \mathbb{P}^{1}$ over $B$. In particular, in case (b) the proper transforms $B_{1}^{\prime}$ and $B_{2}^{\prime}$ of $B_{1}$ and $B_{2}$ in 
$W$ are disjoint (since $B \times\{\infty\}$ and $B \times\{0\}$ are). By Lemma 4.3(2) the dual graph of every singular fiber of the natural morphism $\kappa: W \rightarrow B$ is contractible to the minimal linear subgraph joining two vertices meeting $B_{1}^{\prime}$ and $B_{2}^{\prime}$ respectively. Making all such contractions one gets a morphism $W \rightarrow \tilde{X}$. It remains to note that the morphism $W \rightarrow B \times \mathbb{P}^{1}$ must factor through $W \rightarrow \tilde{X}$ since one wants to keep the proper transforms of $B_{1}^{\prime}$ and $B_{2}^{\prime}$ disjoint. That is, $\varphi=\psi \circ \chi$ where $\psi: \tilde{X} \rightarrow B \times \mathbb{P}^{1}$ is a birational morphism over $B$ and $\chi: \hat{X} \rightarrow \tilde{X}$ is the birational map (that factors through $W$ ).

The image of $\hat{\nu}$ under isomorphism $\hat{X}^{*} \simeq Y^{*}$ yields a complete vector field $\nu_{0}$ on $Y^{*} \backslash B^{*} \times\{\infty\} \simeq B^{*} \times \mathbb{C}_{x}$ tangent to the fibers of the natural projection onto $B^{*}$. Hence, in case (a) the restriction of the field $\nu_{0}$ to every fiber must be proportional to $\mu=\partial / \partial x$ and, thus, there is a regular function $p$ on $B^{*}$ for which $\nu_{0}=p \mu$. The extension $p$ to $B$ is the desired rational function. In case (b) the argument about existence of $p$ is similar but with $\mu=x \partial / \partial x$ which yields (1).

By Proposition 6.1 and Remark 6.2 in case (b) $\mu$ induces a semi-simple field $\tilde{\mu}$ on $\tilde{X}$ whose restriction on any fiber $\operatorname{pr}^{-1}(b)$ of the natural projection $\mathrm{pr}: \tilde{X} \rightarrow B$ has zeros only at the set $Z_{b}$ of double points of the curve $\tilde{B}_{1} \cup \mathrm{pr}^{-1}(b) \cup \tilde{B}_{2}$ where $\tilde{B}_{i}$ is the proper transform of $B_{i}$. By construction the image $I$ of the exceptional divisor $F$ of the morphism $W \rightarrow \tilde{X}$ does not meet $Z_{b}$ for any $b \in B$. That is, $\tilde{\mu}$ does not vanish at any point of $I$ and, therefore, $\tilde{\mu}$ induces a rational vector field on $W$ with poles on $F$. In particular, the restriction of this rational vector field to any fiber of $\kappa: W \rightarrow B$ has a finite number of zeros which yields $(2)$.

Assume that for some $b \in f(X)$ the fiber $\kappa^{-1}(b)$ has a nonlinear dual graph, or, equivalently, $F \cap \kappa^{-1}(b)$ is not empty. The proper transform of $F \cap \kappa^{-1}(b)$ in $\hat{X}$ is not contained in $\hat{D}$ because of pseudo-minimality assumption. That is, there is a component $C$ in $F \cap \kappa^{-1}(b)$ whose proper transform $\hat{C}$ in $\hat{X}$ meets $X$. Hence, $\left.\hat{\mu}\right|_{X}$ is not regular because of poles on $\hat{C}$. Thus, for regularity one needs $\chi$ to be an isomorphism which yields (3).

We need to consider two essentially different cases: when $f: X \rightarrow B$ is surjective and when it is not. As the next claim shows the assumption that there is a vector field $\nu$ tangent to fibers of a $\mathbb{C}$ - and $\mathbb{C}^{*}$-fibration in Setting 6.3 is automatically fulfilled in the non-surjective case.

Proposition 6.5. Let $f: X \rightarrow B$ be a non-surjective morphism from a smooth semiaffine surface $X$ into $B$ with general fibers isomorphic to $\mathbb{C}$ or to $\mathbb{C}^{*}$. Then there is a complete algebraic vector field tangent to the fibers of $f$. Furthermore, one can choose this field so that it does not vanish on a given general fiber $E=f^{-1}\left(b_{0}\right)$ of $f$.

Proof. Suppose first that $f$ is an untwisted $\mathbb{C}^{*}$-fibration (resp. $\mathbb{C}$-fibration). As we showed in the proof of Lemma 6.4 for every regular value $b_{0} \in B$ of $f$ there is a Zariski neighborhood $B^{*} \subset f(X) \subset B$ for which $Z=f^{-1}\left(B^{*}\right)$ is naturally isomorphic to $B^{*} \times \mathbb{C}^{*}\left(\right.$ resp. $B^{*} \times \mathbb{C}$ ) over $B^{*}$. Vector field $\hat{\mu}$ from Lemma 6.4 may have poles on $X \backslash Z$. However, since $f(X)$ is affine one can choose a regular function $h$ on $f(X)$ with prescribed orders of zeros at points of $f(X) \backslash B^{*}$ so that $h \hat{\mu}$ yields a regular complete algebraic vector field on $X$ tangent to the fibers of $f$. Choosing $h$ so that $h\left(b_{0}\right) \neq 0$ we get the second statement. 
In the twisted case consider a proper extension $\hat{f}: \hat{X} \rightarrow B$ of $f$ to an SNC-completion $\hat{X}=X \cup \hat{D}$ of $X$. Then $\hat{D}$ has the only one irreducible component $B_{0}$ on which the restriction of $\hat{f}$ is not a constant. Furthermore, $p:=\left.\hat{f}\right|_{B_{0}}$ makes $B_{0}$ a ramified double cover of $B$. Hence, one has a $\mathbb{Z}_{2}$-action $\alpha$ on $B_{0}$ for which $B \simeq B_{0} / \alpha$. Let $X_{0}=X \times_{B} B_{0}$ and $q: X_{0} \rightarrow B_{0}, r: X_{0} \rightarrow X$ be the induced morphisms. Since $r$ makes $X_{0}$ a ramified double cover of $X$ we have again a $\mathbb{Z}_{2}$-action $\beta$ on $X_{0}$ for which $X=X_{0} / \beta$. Consider an $\alpha$-invariant Zariski dense open subset $B_{0}^{*} \subset p^{-1}(f(X)) \subset B_{0}$ for which $q^{-1}\left(B_{0}^{*}\right)$ is naturally isomorphic to $B_{0}^{*} \times \mathbb{C}^{*}$ where the second factor is equipped with a coordinate $x$. Then the restriction of $\beta$ to $q^{-1}\left(B_{0}^{*}\right)$ is given by $\beta(b, x)=(\alpha(b), g(b, x))$ where $(b, x) \in B_{0}^{*} \times \mathbb{C}^{*}$ and for a fixed $b$ the function $g(b, x)$ is a coordinate on $\mathbb{C}^{*}$. That is, $g(b, x)$ coincides either with $e(b) x$ or with $e(b) / x$ with $e(b)$ being a non vanishing regular function on $B_{0}^{*}$. However, the first possibility must be disregarded because we deal with a twisted case.

In order to construct a complete algebraic vector field on $X$ tangent to the fibers of $f$ on $X$ it suffices to construct a complete algebraic vector field on $(p \circ q)^{-1}(f(X))$ tangent to the fibers of $q$ and such that its restriction to $q^{-1}\left(B_{0}^{*}\right)$ is $\beta$-invariant. Note that the field $x \frac{\partial}{\partial x}$ is mapped to $-x \frac{\partial}{\partial x}$ under automorphism $x \rightarrow e(b) / x$ of $\mathbb{C}^{*}$. Hence, for every function regular function $h$ on $B_{0}^{*}$ that is $\alpha$-antisymmetric the field $h x \frac{\partial}{\partial x}$ is invariant with respect to the $\beta$-action. Choosing this function $h$ on $B_{0}$ so that its extension to the affine curve $p^{-1}(f(X))$ has zeros of sufficiently high order at points of $p^{-1}(f(X)) \backslash B_{0}^{*}$ we guarantee the regular extension of this field to $(p \circ q)^{-1}(f(X))$ which yields the first statement. For the second statement choose $B_{0}^{*}$ so that it contains $p^{-1}\left(b_{0}\right)$ and require that $h$ does not vanish on this set. Hence, we are done.

In particular, the presence of $\mathbb{C}$ - and $\mathbb{C}^{*}$-fibrations in Theorem 5.8 implies the following.

Corollary 6.6. Every normal semi-affine surface $X$ with a complete algebraic vector field which does not possess a rational first integral has automatically an open orbit.

Lemma 6.7. Let $\hat{\nu}$ and $\hat{f}: \hat{X} \rightarrow B$ be as in Setting 6.3 and $f: X \rightarrow B$ be surjective. Then

(i) $f$ is an untwisted $\mathbb{C}^{*}$-fibration;

(ii) every singular fiber of $\hat{f}$ has a linear dual graph $C_{1}+\ldots+C_{n}+C+C_{n^{\prime}}^{\prime}+\ldots+C_{1}^{\prime}$ where $n, n^{\prime} \geq 1, C$ is the only $(-1)$-vertex of this fiber (i.e., $C_{i} \cdot C_{i}$ and $C_{i}^{\prime} \cdot C_{i}^{\prime} \leq-2$ ), $C_{i} \subset \hat{D}$ and $C_{i}^{\prime} \subset \hat{E}$ while $C$ meets both $X$ and $\hat{D}$;

(iii) the field $\hat{\nu}$ (and, therefore, $\nu$ ) is semi-simple.

Proof. Assume that contrary to (i) we deal with case (a) from Setting 6.3. Let $\varphi$ : $\hat{X} \rightarrow B \times \mathbb{P}^{1}, \hat{\mu}$ and $p$ be as in Lemma 6.4 , and let $B^{*}$ and $\hat{X}^{*} \simeq B^{*} \times \mathbb{P}^{1}$ be as in the proof of that Lemma. In particular, $p$ is regular on $B^{*}$ and $\hat{\nu}=\hat{f}^{*}(p) \hat{\mu}$ is locally nilpotent on $\hat{X}^{*} \backslash B_{0} \simeq B^{*} \times \mathbb{C}_{x}$ ( since $\hat{\nu}=p \frac{\partial}{\partial x}$ ). That is, the flow of this complete vector field $\hat{\nu}$ induces an algebraic $\mathbb{C}_{+}$-action on $\hat{X}^{*} \backslash B_{0}$ and, therefore, on $X=\hat{X} \backslash \hat{D}$. However, the algebraic quotient $X / / \mathbb{C}^{+}$must be affine (since $X$ is a semi-affine surface) while the surjectivity of $\left.f\right|_{X}$ implies that this quotient is $B$. Hence, since $B$ is complete we have to disregard case (a). 
Suppose now that we are in case (b) and use the notation from Lemma 6.4. By surjectivity of $\left.f\right|_{X}$ every singular fiber $\hat{f}^{-1}(b)$ contains an irreducible component $C$ that meets $\hat{D}$ but is not contained in $\hat{D}$. By Lemma $6.4 \hat{\mu}$ does not vanish identically on $C$. Hence, $p$ has no pole in $b$ since the field $\hat{\nu}=\left.\hat{f}^{*}(p) \hat{\mu}^{*}\right|_{X}$ is regular. The absence of poles on the complete curve $B$ means that $p$ is constant and, thus, we can suppose that $\hat{\mu}=\hat{\nu}$.

This implies in particular that $\hat{\mu}$ is regular on $\hat{X} \backslash \hat{D}$. By Lemma 6.4(3) the fibers of $\hat{f}$ are linear chains of rational curves. Note also that $\hat{D} \cup \hat{E}$ is the union of $B_{1}, B_{2}$, and all irreducible components of singular fibers with exception of components similar to $C$. Therefore, $B_{1}$ and $B_{2}$ belong to different connected components of $\hat{D} \cup \hat{E}$. Recall that $B_{1} \subset \hat{D}$. Since $\hat{D}$ is connected by the Lefschetz theorem, we see that the connected component containing $B_{1}$ (resp. $B_{2}$ ) coincides with $\hat{D}$ (resp. $\hat{E}$ ).

Assume that $n=0$, i.e., $C$ meets section $B_{1}$ which implies that $C$ is irreducible in the fiber $\hat{f}^{*}(b)$. By Lemma 4.3 and pseudo-minimality of $\hat{E}$ one has $n^{\prime}=0$ contrary to the fact that $\hat{f}^{-1}(b)$ is singular. Thus, $n \geq 1$ and similarly $n^{\prime} \geq 1$ which yields (ii).

It remains to exclude the case of twisted $\mathbb{C}^{*}$-fibration, i.e the case when $\hat{D}$ contains a double section $B_{0}$. Let us replace $\hat{f}: \hat{X} \rightarrow B$ by the natural morphism $\hat{X} \times{ }_{B} B_{0} \rightarrow B_{0}$ and also replace $\hat{\nu}, X$, and $\hat{D}$ by their lifts to $\hat{X} \times_{B} B_{0}$. Then two sections of the modified morphism $\hat{f}: \hat{X} \rightarrow B$ are contained in $\hat{D}$ contrary to the argument before, i.e., we have (i) and (ii).

Lemma 6.4 (3) and the equality $\hat{\nu}=\hat{\mu}$ imply (iii) which concludes the proof.

Remark 6.8. (1) It follows from the proof that $\hat{E}$ is connected. Thus, the Remmert reduction $X_{0}$ of $X$ has only one singularity which is automatically a fixed point of an elliptic $\mathbb{C}^{*}$-action associated with $\nu$. Hence, Lemma 6.7 can be also obtained from the description of normal $\mathbb{C}^{*}$-singularities according to $[35,36]$. It can be extracted as well from the DPD-presentation for $\mathbb{C}^{*}$-surfaces due to Flenner and Zaidenberg [14].

(2) Furthermore, if $X_{0}$ is smooth then the Luna slice theorem implies that $X_{0} \simeq \mathbb{C}^{2}$ [30].

(3) In the case when $X_{0}$ is not smooth consider the field $\nu_{0}$ induced by $\nu$ on $X_{0}$ and the rational first integral $f_{0}$ induced by $f$. One can see that the surjectivity of $f: X \rightarrow B$ is equivalent to the fact that $f_{0}$ is not regular on $X_{0}$.

Setting 6.9. Suppose we are in Setting 6.3 and let $g: X \rightarrow B^{\prime}$ be another $\mathbb{C}$ - or $\mathbb{C}^{*}$ fibration on $X$ over a complete curve $B^{\prime}$ such that a general fiber of $g$ is not contained in a general fiber of $f$ and vice versa. Let $\hat{g}: \hat{X}^{\prime} \rightarrow B^{\prime}$ be a proper extension of $g$ to an SNC-completion of $X$ by a pseudo-minimal divisor $\hat{D}^{\prime}$.

Proposition 6.10. Assume the Setting 6.9. Then $B$ (and, therefore, $B^{\prime}$ ) is a rational curve and $X$ is a rational surface. Furthermore, if $f: X \rightarrow B$ is surjective then either

(1) the dual graph of $\hat{D}$ (and, therefore, of $\hat{E}$ ) is linear, or

(2) the dual graph of $\hat{D}$ is of form

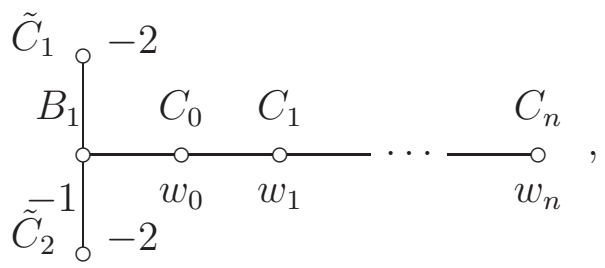


where $w_{i} \leq-2$ for $i \geq 0$. In particular, in (2) $\hat{f}$ has three singular fibers $\hat{f}^{-1}\left(b_{0}\right), \hat{f}^{-1}\left(b_{1}\right)$, and $\hat{f}^{-1}\left(b_{2}\right)$ containing $C_{0}, \tilde{C}_{1}$, and $\tilde{C}_{2}$ respectively.

Moreover, $g$ is not surjective.

Proof. By assumption the restriction of $\left.f\right|_{F}: F \rightarrow B$ to a general fiber $F$ of $g$ is not constant. Hence, $B$ is rational since $F$ is rational. This implies also that $X$ is rational because $f$ is a $\mathbb{C}$ - or $\mathbb{C}^{*}$-fibration.

Assume that $f$ is surjective and $\hat{f}$ has three or more singular fibers or equivalently that the dual graph of $\hat{D}$ is not linear. By Lemma $6.7 B_{1}$ is the only branch point of this graph and the weight of any other vertex is at most -2 . The identical automorphism of $X$ extends to a rational map $\hat{X} \rightarrow \hat{X}^{\prime}$, i.e., there is a reconstruction of the dual graph $\Gamma$ of $\hat{D}$ into a dual graph $\Gamma^{\prime}$ of $\hat{D}^{\prime}$.

By pseudo-minimality the weights of linear vertices of $\Gamma^{\prime}$ are at most -2 . Hence, Corollary 3.5 implies that if $\Gamma^{\prime}$ is minimal then it coincides with $\Gamma$ and otherwise it is obtained from $\Gamma$ by a sequence of inner and outer blowing up. In particular, $\Gamma^{\prime}$ cannot contain a linear 0 -vertex and if it has a branch vertex of weight -1 then it is a proper transform of $B_{1}$.

However, if $\hat{g}: X \rightarrow B^{\prime}$ is not surjective then by Proposition 4.6 there must be either a subgraph of type $\Gamma_{*}$ from Proposition $4.6(3 \mathrm{~b})$ or a 0 -vertex in $\Gamma^{\prime}$. In combination with the previous argument Proposition 4.6 leads to the graph in (2).

Thus, it remains to consider the case when $g: X^{\prime} \rightarrow B^{\prime}$ is surjective. Let us derive a contradiction by showing that the fibrations $\hat{f}$ and $\hat{g}$ must coincide in this case. As before we see that $\Gamma^{\prime}$ is obtained from $\Gamma$ by a sequence of blowing-ups. Hence, by Corollary 3.5 of $\hat{D}$ we can suppose that $\hat{X}=\hat{X}^{\prime}$.

Let $F$ (resp. $G$ ) be a general fiber of $\hat{f}$ (resp. $\hat{g}$ ). It suffices to show that $F$ is equivalent to $G$ in $H^{2}(\hat{X}, \mathbb{Z})$ (because the linear systems $|F|$ and $|G|$ induce $\hat{f}$ and $\hat{g}$ respectively). Let $S$ (resp. $S^{\prime}$ ) be the elements of this cohomology group corresponding to the vertices of the dual graphs of $\hat{E} \backslash B_{2}$ (resp. $\hat{D} \backslash B_{1}$ ). Since $\hat{X}$ is contractible to a surface ruled over $B$ we see that the elements of $S$ and $S^{\prime}$ together with $F$ and $B_{2}$ form a basis of $H^{2}(\hat{X}, \mathbb{Z})$. In particular, in this cohomology group $G=k F+l B_{2}+M+M^{\prime}$ where $M$ (resp. $M^{\prime}$ ) is an integer linear combination of elements from $S$ (resp. $S^{\prime}$ ). Note that the restriction of the intersection form to $S$ (resp. $S^{\prime}$ ) is negative definite by the Zariski lemma [4, page 90]. By the same reason $F \cdot C_{i}=F \cdot C_{j}^{\prime}=G \cdot C_{i}=G \cdot C_{j}^{\prime}=0$. Hence, $\left(M^{\prime}\right)^{2}=G \cdot M^{\prime}=0$ which implies that $M^{\prime}$ is the zero divisor. Since $G \cdot B_{1}=1$ we have $k=1$ and $G=F+l B_{2}+M$. Then equalities $B_{2} \cdot G=1, G \cdot M=0$ and $(G)^{2}=0$ imply that

$$
l\left(B_{2}\right)^{2}+B_{2} \cdot M=0, M^{2}+l B_{2} \cdot M=0, \text { and } M^{2}+l^{2}\left(B_{2}\right)^{2}+2 l B_{2} \cdot M+2 l=0 .
$$

In turn the last three equalities yield $l=0$ and, therefore, $M^{2}=0$. The semi-negativity implies that $M=0$ and $G=F$ which is the desired conclusion.

Proposition 6.11. Let us be in Setting 6.9 and $f: X \rightarrow B$ be surjective. Suppose also that $\hat{D}$ has a dual graph different from the graph in Proposition 6.10 (2). Then the Remmert reduction $X_{0}$ of $X$ is a normal toric surface.

Proof. Since $\hat{D}$ is the boundary divisor of a semi-affine surface the Nakai-Moishezon criterion implies that $B_{1}^{2} \geq-1$ and, furthermore, if $B_{1}^{2}=-1$ then $\hat{D}$ can be contracted 
to a curve which contains an irreducible component of zero weight and some other components of negative weight. If $B_{1}^{2}>0$ then after several inner blowing-ups we can make its weight equal to zero. In both cases (and also in the case of $B_{1}^{2}=0$ ) one of the end vertices of the dual graph of the resulting curve $\tilde{D}$ is of negative weight and it corresponds to the end-vertex $C_{n}$ of $\hat{D}$ which is automatically contained in some singular fiber $\hat{f}^{-1}(b)$. Let $C_{1}+\ldots+C_{n}+C+C_{n^{\prime}}^{\prime}+\ldots+C_{1}^{\prime}$ be the dual graph of $\hat{f}^{-1}(b)$ as in Lemma 6.7. Then the dual graph of $\tilde{D}$ is of form $F_{1}+F_{2}+\ldots+F_{k}+C_{n}$ and furthermore, using elementary transformations from Proposition 3.3 we can suppose that $F_{1}$ is of zero weight. In particular, $F_{1}$ generates a $\mathbb{P}^{1}$-fibration $\tilde{f}: \tilde{X} \rightarrow \mathbb{P}^{1}$ on the resulting surface $\tilde{X}$. Note that by construction $F_{2}$ (which may be equal to $C_{n}$ ) is a section of this fibration and the only singular fiber $\tilde{f}^{-1}(a)$ of $\tilde{f}$ is the union $G$ of $\hat{E}$ and the components $F_{3}, \ldots, F_{k}, C_{n}$, and $C$. Indeed $G$ is connected and does not meet $F_{1}$ (i.e., it is contained in a fiber of $\tilde{f}$ ), and there is no complete irreducible curve different from a component of $G$ that does not meet both $F_{1}$ and $F_{2}$ (which would be the case if $G$ is not the whole fiber or in the case of another singular fiber). Furthermore, since $X=\hat{X} \backslash \hat{D}=\tilde{X} \backslash \tilde{D}$ and the dual graph of $\hat{D}$ (or $\tilde{D}$ ) is linear the Remmert reduction $X_{0}$ of $X$ is a normal Gizatullin surface and in terminology of [12] the curve $\tilde{D} \cup \tilde{f}^{-1}(a)$ is called the extended boundary divisor. A normal Gizatullin surface (say $X_{0}$ ) is toric if and only if the dual graph of the extended boundary divisor for its minimal resolutions of singularities (say $X$ ) is linear [12, Lemma 2.20]. This is exactly the case of the dual graph of $\tilde{D} \cup \tilde{f}^{-1}(a)$ and we are done.

Proposition 6.12. Let us be in Setting 6.9 and $f: X \rightarrow B$ be surjective. Suppose also that the dual graph of $\hat{D}$ is as in Proposition 6.10 (2). Then

(1) $X$ is a surface with an open orbit;

(2) the flow of $\nu$ preserves the fibers of $g$;

(3) the Remmert reduction $X_{0}$ of $X$ is not generalized Gizatullin unless $n=0$ and $w_{0}=-2$ in which case the dual graph of the curve $\hat{E}$ has the following form

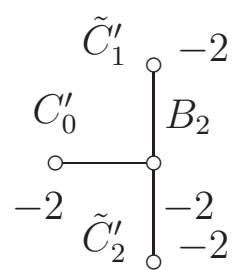

whence the dual graph of every singular fiber $\hat{f}^{-1}\left(b_{i}\right)$ coincides with $[[-2,-1,-2]]$ for $i=0,1,2$. Furthermore, such a surface $X$ is unique up to isomorphism.

Proof. By Proposition 6.5 there is a complete algebraic vector field $\mu$ tangent to the fibers of $g$. Hence, $\nu$ is not proportional to $\mu$ which yields (1).

By Lemma $6.7 \hat{\nu}$ (resp. $\nu$ ) is a semi-simple field on $\hat{X}$ (resp. $X$ ). In particular, the flow $\Phi_{t}$ of $\hat{\nu}$ preserves the curve $F_{\infty}=\tilde{C}_{1} \cup B_{1} \cup \tilde{C}_{2}$ which is the fiber of $\hat{g}$. Thus, for any time parameter $t$ and every other fiber $F$ of $\hat{g}$ the image $\Phi_{t}(F)$ does not meet $F_{\infty}$ which implies that $\hat{g}$ is constant on $\Phi_{t}(F)$, i.e., it is again a fiber of $\hat{g}$. This yields $(2)$.

By Lemma 6.7 the dual graph of $\hat{f}^{-1}\left(b_{1}\right)$ is

$$
\tilde{C}_{1}+\tilde{C}+\tilde{C}_{n^{\prime}}^{\prime \prime}+\ldots+\tilde{C}_{1}^{\prime \prime}
$$


where $\tilde{C}$ is the only $(-1)$-vertex, any other weight is at most -2 , and $\tilde{C}_{1}^{2}=-2$. Since this fiber of a $\mathbb{P}^{1}$-fibration must be contractible to a 0 -vertex, contracting $\tilde{C}$ and $\tilde{C}_{1}$ consequently we see that $n^{\prime}=1$ and $\left(C_{1}^{\prime \prime}\right)^{2}=-2$. The same is valid for the fiber $\hat{f}^{-1}\left(b_{2}\right)$, and in the case of $n=0$ and $w_{0}=-2$ it is also true for $\hat{f}^{-1}\left(b_{0}\right)$.

Assume that $w_{0} \leq-3$. Consider the dual graph

$$
C_{0}+C_{1}+\ldots+C_{n}+C+C_{n^{\prime}}^{\prime}+\ldots+C_{1}^{\prime}
$$

of $\hat{f}^{-1}\left(b_{0}\right)$. Then the connected curve

$$
G=C_{1}+\ldots+C_{n}+C+C_{n^{\prime}}^{\prime}+\ldots+C_{1}^{\prime}+B_{2}+\tilde{C}_{1}^{\prime}+\tilde{C}_{2}^{\prime}
$$

is a fiber of $\hat{g}$ since it contains all complete curves in $\hat{X}$ that do not meet the fiber $F_{\infty}$ or the section $C_{0}$. Note that $B_{2}$ is branch point of the dual graph of $G$ and all branches but

$$
\mathcal{B}=C_{1}+\ldots+C_{n}+C+C_{n^{\prime}}^{\prime}+\ldots+C_{1}^{\prime}
$$

are non-contractible (because we know already that the weights of $\tilde{C}_{1}^{\prime}$ and $\tilde{C}_{2}^{\prime}$ are -2 ). Thus, the latter must be contractible since any fiber is contractible to a 0 -vertex and furthermore, after this contraction the weight of $B_{2}$ must become -1 in the graph $[[-2,-1,-2]]$. Hence, as in the proof of Proposition 4.6 we conclude that $B_{2}$ is a multiple component of $\hat{g}$ and, therefore, each component of $\mathcal{B}$ is also multiple. In particular, $C$ is a multiple component of $g$, i.e., $C \cap X$ is a singular fiber of $g$.

Suppose that $\eta$ is a complete algebraic vector field without a rational first integral. By Theorem 5.8 there must a $\mathbb{C}^{*}$ - or $\mathbb{C}$-fibration over $\mathbb{C}$ such that its fibers are transformed into each other by the flow of $\eta$ and $g$ is the only candidate for this fibration (since its minimal extension $\hat{g}$ to a $\mathbb{P}^{1}$-fibration is determined uniquely by the form of $\hat{D})$. However, by Lemma 8.3 the curve $C \cap X$ is preserved by this flow.

Similarly, if $C \cap X$ is a component of a fiber of a rational first integral of some complete vector field it is preserved by the flow of this field (in particular this is true for $\nu$ and $\mu$ ). If we have $w_{0} \leq-3$ or $n \geq 1$ then $g$ is the unique $\mathbb{C}^{*}$ - or $\mathbb{C}$-fibration on $X$ and, thus, the curve $C \cap X$ is preserved by all complete vector fields. Since the image of $C \cap X$ in $X_{0}$ is a curve $X_{0}$ cannot be generalized Gizatullin when $w_{0} \leq-3$. or $n \geq 1$.

Thus, we have the desired form of the dual graph of $\hat{E}$ except for the fact that the weight $k$ of $B_{2}$ is still unknown. Note first that $k \leq-2$ since otherwise the intersection matrix of $\hat{E}$ is not negative definite contrary to the Grauert criterion of contractibility [4, Theorem III.2.1]. Then contraction of fibers $\hat{f}^{-1}\left(b_{0}\right), \hat{f}^{-1}\left(b_{1}\right)$, and $\hat{f}^{-1}\left(b_{2}\right)$ to 0 vertices transforms $\hat{X}$ into a Hirzebruch surface $\hat{X}^{\prime}$ with proper transforms $B_{1}^{\prime}$ and $B_{2}^{\prime}$ of $B_{1}$ and $B_{2}$ as disjoint sections. Their weights $k_{1}$ and $k_{2}$ depends on the choice of contraction of these three fibers but in any case $k_{1}+k_{2}=-1+k+3=k+2 \leq 0$. Since these sections are disjoint it follows from [21, page 518] that one of them is of negative weight (say $-m$ ) and the weight of the other is at least $m$ which yields $k=-2$ and we are done with the form of the graph of $E$.

Furthermore, we see now that $\hat{X}^{\prime}$ can be chosen as $\mathbb{P}^{1} \times \mathbb{P}^{1}$ and $\hat{X}$ is obtained from $\hat{X}^{\prime}$ by some standard blowing up in three fibers of a natural morphism $\hat{X}^{\prime} \rightarrow \mathbb{P}^{1}$. Since the group of automorphisms of $\mathbb{P}^{1}$ acts transitively on the triples of distinct points we get the claim about uniqueness. 
Example 6.13. Let $X_{0}$ be from Proposition 6.12. Then its singularity (whose minimal resolution is given by the graph of $\hat{E}$ ) is a du Val singularity of type $D_{4}$ where a singularity of type $D_{n+1}$ is locally isomorphic to the hypersurface $y x^{2}+y^{n}+z^{2}=0$ in $\mathbb{C}_{x, y, z}^{3}$. In fact for $n=3$ one can see that $X_{0}$ is globally isomorphic to the hypersurface $y\left(x^{2}+y^{2}\right)+z^{2}=0$. The elliptic $\mathbb{C}^{*}$-action on it is given by $(x, y, z) \rightarrow\left(\lambda^{2} x, \lambda^{2} y, \lambda^{3} z\right)$ for $\lambda \in \mathbb{C}^{*}$ and it corresponds to a semi-simple field $\sigma$. Three $\mathbb{C}^{*}$-fibrations associated with the three different strings $[[-2,-1,-2]]$ in the graph of $\hat{D}$ are given by the functions $y, x+\sqrt{-1} y$, and $x-\sqrt{-1} y$. By Proposition 6.5 there are complete algebraic vector fields $\sigma_{1}, \sigma_{2}, \sigma_{3}$ on the hypersurface tangent to the fibers of these functions respectively. Note that the only curve tangent to both $\sigma$ and $\sigma_{1}$ is given by $y=0$, i.e this curve is invariant under their flows. However, it is not invariant under the flows of $\sigma_{2}$ or $\sigma_{3}$ and, hence, one can check that the natural action of $\operatorname{AAut}_{\text {hol }}\left(X_{0}\right)$ has the smooth part of $X_{0}$ as an open orbit. In particular, $X_{0}$ is generalized Gizatullin (another proof of this fact will be considered in the last section) and, hence, it is the surface described in Proposition $6.12(3)$. For $n \geq 4$ there is only one regular $\mathbb{C}^{*}$-fibration on the corresponding surface (given by function $y$ ) which is, therefore, not a generalized Gizatullin surface, only a surface with an open orbit.

Theorem 6.14. Let $X_{0}$ be a generalized Gizatullin surface such that for a complete algebraic vector field $\nu_{0}$ on $X_{0}$ there is a surjective rational first integral $f_{0}: X_{0} \rightarrow B$ into a complete curve $B$. Then

(1) either $X_{0}$ is toric (and in particular a Gizatullin surface) or $X_{0}$ is the Remmert reduction of $X$ from Proposition 6.12;

(2) up to a constant nonzero factor $\nu_{0}$ is semi-simple.

Proof. Statement (2) follows from 6.7(iii). For (1) consider a birational morphism $X \rightarrow X_{0}$ from a semi-affine surface $X$ such that $f_{0}$ induces a surjective morphism $f: X \rightarrow B$ with general fibers isomorphic to $\mathbb{C}$ or $\mathbb{C}^{*}$. If a complete algebraic vector field $\mu_{0}$ on $X_{0}$ non-proportional to $\nu_{0}$ has also a rational first integral we can suppose that it induces a similar morphism $g: X \rightarrow B^{\prime}$. Then Propositions 6.11 and 6.12 imply the desired conclusion.

If $\mu_{0}$ does not have a rational first integral then by Theorem 5.8 there is a morphism $g_{0}: X_{0} \rightarrow B^{\prime}$ with general fibers isomorphic to $\mathbb{C}$ or $\mathbb{C}^{*}$. Since $f_{0}$ has indeterminacy points its general fibers are different from the general fibers of $g_{0}$ and, therefore, the fibration $g: X \rightarrow B^{\prime}$ (induced by $g_{0}$ ) is different from $f: X \rightarrow B$. By Proposition 6.5 there is a complete vector field tangent to the fibers of $g$ and we are done again by Proposition 6.11.

Now we are in the position to prove our second important result from the introduction, which in the case of $\mathbb{C}^{2}$ can be extracted from [8].

Proof. (of Theorem 1.5) Assume that $X$ has no open orbit, i.e., $\nu$ must have a rational first integral $f: X \rightarrow B$ by Proposition 6.5. Then we have (1).

For (2) consider a surface $X$ with an open orbit. Because of Proposition 6.5 we can suppose again that $\nu$ has a rational first integral. If such an integral is surjective then by Theorem 6.14 we may deal either with a toric surface or with the surface from Proposition 6.12. In the latter case the second statement of Proposition 6.12 yields 
the desired regular function $f: X \rightarrow \mathbb{C}$. In the former case the existence of such $f$ was established in [27] or it can be extracted from explicit description of algebraic $\mathbb{C}^{*}$-actions on Gizatullin surfaces in [12]. Now suppose that every complete algebraic vector field on $X$ has a regular rational first integral. Then such integrals for nonproportional fields lead to different $\mathbb{P}^{1}$-fibrations on a completion of $X$ satisfying the assumption of Proposition 6.10 whence $X$ is rational. Hence, for any $\mathbb{C}$ - or $\mathbb{C}^{*}$-fibration $X \rightarrow B$ one has $B \simeq \mathbb{C}$. In any case this regular rational first integral for $\nu$ can be viewed as a desired $f: X \rightarrow \mathbb{C}$ in (2) and we are done.

\section{Dual graphs of CURVes in Rational surfaces}

The aim of this section is the following.

Proposition 7.1. (1) Let $\Gamma$ be a graph as in Theorem 1.4 (3) (resp. (5)) such that $w_{0} \geq$ 0. Then $\Gamma$ is the dual graph of an SNC curve (with rational irreducible components) contained in a smooth complete rational surface if and only if the assumption ( $\alpha$ ) (resp. ( $\beta$ )) from Theorem 2.14 holds.

(2) Let $\Gamma$ be a graph as in Theorem 1.4 (2a). Then $\Gamma$ is the dual graph of an SNC curve (with rational irreducible components) contained in a smooth complete rational surface if and only if the assumption $(\gamma)$ from Theorem 2.14 holds.

The proof requires some preparations.

Lemma 7.2. Let $C_{0}$ be a smooth rational curve in a smooth complete rational surface $\bar{X}$ admitting a $\mathbb{P}^{1}$-fibration $\bar{f}: \bar{X} \rightarrow \mathbb{P}^{1}$ such that the map $\left.\bar{f}\right|_{C_{0}}: C_{0} \rightarrow \mathbb{P}^{1}$ has degree 2. Then there is a regular birational map $\iota: \bar{X} \rightarrow \hat{X}$ to Hirzebruch surface $\hat{X}$ such that its restriction $\left.\iota\right|_{C_{0}}: C_{0} \rightarrow \hat{C}_{0}:=\iota\left(C_{0}\right)$ is an isomorphism and $\bar{f}$ factors through a natural projection $\hat{f}: \hat{X} \rightarrow \mathbb{P}^{1}$.

Proof. By the Riemann-Hurwitz formula there are only two points $b_{0}, b_{1} \in \mathbb{P}^{1}$ such that $F_{i}=f^{-1}\left(b_{i}\right), i=0,1$ meets $C_{0}$ at one point. Since the degree of $\left.\bar{f}\right|_{C}: C \rightarrow \mathbb{P}^{1}$ is 2 every other fiber $F$ of $\bar{f}$ meets $C_{0}$ transversely at two points and the components of $F$ that meet $C_{0}$ are reduced in $\bar{f}^{*}(b)$ (there at most two of them, say $E_{1}$ and $E_{2}$ ). By Lemma 4.3 we can contract $F$ first to a linear chain whose end points are $E_{1}$ and $E_{2}$ and then contract it further to $E_{1}$. Note that this procedure does not produce the singularities on the image of $C_{0}$ since we contract consequently $(-1)$-curves which are transversal to (the proper transform of) $C_{0}$ and which meet it at most at one point. By Theorem 4.2 we can contract also $F_{0}$ and $F_{1}$ to 0 -vertices which yields the desired Hirzebruch surface. By construction $\left.\iota\right|_{C_{0}}: C_{0} \rightarrow \hat{C}_{0}$ is injective and it remains to show that $\hat{C}_{0}$ is smooth over $b_{0}$ and $b_{1}$.

If more than one irreducible component of $F_{0}$ meets $C_{0}$ then we can argue as in the case of a general fiber $F$. Thus, we can suppose that only one irreducible component of $F_{0}$ meets $C_{0}$. If it is not transversal to $C_{0}$ then its multiplicity in $\bar{f}^{*}\left(b_{0}\right)$ is 1 and we can contract the rest of the fiber $F_{0}$ to it without affecting $C_{0}$. Thus, we can suppose that this component meets $C_{0}$ transversely and its multiplicity in $\bar{f}^{*}\left(b_{0}\right)$ is 2 . Then again by Lemma $4.3(3)$ we can contract $F_{0}$ to a chain $\tilde{C}_{1}+E+\tilde{C}_{2}=[[-2,-1,-2]]$ without producing a singularity and with $E$ meeting the proper transform of $C_{0}$ transversely. Note that contracting consequently $E$ and $\tilde{C}_{1}$ we do not produce a singularity either by 
the same transversality argument. One can deal with $F_{1}$ in the similar manner which concludes the proof.

Proposition 7.3. Let $C$ be a smooth rational curve in a Hirzebruch surface $\hat{X}=\mathbb{F}_{n}$ such that $\left.\kappa\right|_{C}: C \rightarrow \mathbb{P}^{1}$ has degree 2 where $\kappa: \hat{X} \rightarrow \mathbb{P}^{1}$ is the natural projection. Then $C \cdot C=4$.

Proof. Treating closed curves in $\hat{X}$ as elements of the Néron-Severi group of $\hat{X}$ we recall that this group is generated by a fiber $\hat{F}$ of $\kappa$ and the negative section $S$ of $\kappa$, i.e., $\hat{F}^{2}=0, \hat{F} \cdot S=1$ and $S^{2}=-n$. Since $\hat{F}$ is a smooth rational curve the adjunction formula yields $\left(K_{\hat{X}}+\hat{F}\right) \cdot \hat{F}=-2$ (where $K_{\hat{X}}$ is the canonical divisor). Hence, $K_{\hat{X}}=-2 S+m \hat{F}$ for some $m \in \mathbb{Z}$. On the other hand, by the same reason $\left(K_{\hat{X}}+S\right) \cdot S=-2$ which implies that $m=-2-n$ and $K_{\hat{X}}=-2 S-(n+2) \hat{F}$. Since $\left.\kappa\right|_{C}$ is of degree 2 we have $C=2 S+l \hat{F}$ for some $l \in \mathbb{Z}$. Since $C$ is also smooth rational we have $\left(K_{\hat{X}}+C\right) \cdot C=-2$ which implies that $2(l-n-2)=-2$. Hence, $l=n+1$. Now we have $C^{2}=-4 n+4(n+1)=4$ which concludes the proof.

Corollary 7.4. Let $\Gamma_{0}$ be a graph as in Proposition 4.6 (3b) and $\Gamma$ be a graph containing $\Gamma_{0}$ such that $B_{0}$ is the only neighbor of the chain $C_{1}+E+C_{2}$ in $\Gamma$. Suppose that $\Gamma$ is the dual graph of a SNC-curve in a smooth complete rational surface $\bar{X}$ such that all irreducible components of this curve are rational. Then the weight of $B_{0}$ is at most 2 (and it is exactly 2 when $\Gamma=\Gamma_{0}$ ).

Proof. The chain generates a $\mathbb{P}^{1}$-fibration $\bar{f}: \bar{X} \rightarrow \mathbb{P}^{1}$. By Lemma 7.2 there is a contraction $\iota: \bar{X} \rightarrow \hat{X}$ to a Hirzebruch surface $\hat{X}$ in which the image $C$ of $B_{0}$ has self intersection $w=4$ by Proposition 7.3. Since $\iota$ involves contraction of at least two consequent neighbors of $C$ (say, $E$ and $C_{1}$ ) we see that the weight of $B_{0}$ is at most $4-2=2$ which yields the desired conclusion.

Remark 7.5. For $\hat{X}=\mathbb{F}_{n}$ with $n \geq 2$ a curve $C$ as in Proposition 7.3 does not exist. Indeed, as we saw in the proof $C=2 S+(n+1) F$ and, hence, $C \cdot S=1-n<0$ which is absurd. For the quadric $\mathbb{P}^{1} \times \mathbb{P}^{1}$ such curve $C$ obviously exists (it is enough to consider the standard parabola in $\mathbb{C}^{2} \subset \mathbb{P}^{1} \times \mathbb{P}^{1}$ and it completion in the quadric). Choose a general line $S^{\prime}=b_{1} \times \mathbb{P}^{1}$ and a line $F=\mathbb{P}^{1} \times b_{2}$ such that $F \cap C \cap S$ is a point $a$. Blowing up the quadric at $a$ and contracting the proper transform of $F$ we get a Hirzebruch surface $\mathbb{F}_{1}$ for which the proper transform of $S^{\prime}$ is the negative section. Note that the proper transform of $C$ is still smooth and its natural map to $\mathbb{P}^{1}$ is still of degree 2 , i.e., we have also the desired curve for $\mathbb{F}_{1}$.

Proposition 7.6. Statement (1) of Proposition \%.1 is true.

Proof. Assume $\bar{X}$ is a smooth complete rational surface containing a connected SNCcurve $G$ with rational components such that its dual graph $\Gamma$ is as in Theorem 1.4 (3) or (5). Then $\Gamma$ contains a subgraph $\Gamma_{0}$ as in Proposition 4.6 (3) which contains in turn the chain $\tilde{C}_{1}+E+\tilde{C}_{2}$ generating a $\mathbb{P}^{1}$-fibration $\bar{f}: \bar{X} \rightarrow \mathbb{P}^{1}$. The vertex $C_{0}$ of $\Gamma_{0}$ yields a smooth rational curve such that $\left.\bar{f}\right|_{C_{0}}: C_{0} \rightarrow \mathbb{P}^{1}$ has degree 2 . Making contractions in all fibers of $\bar{f}$ but the one containing the chain we obtain another $\mathbb{P}^{1}$ fibration $\kappa: \hat{X} \rightarrow \mathbb{P}^{1}$ with all fibers but one (say, $F_{0}$ ) irreducible and by Lemma 7.2 we can suppose that the image $B_{0}$ of $C_{0}$ is smooth. That is, the curve $F_{0} \cup B_{0}$ has 
the same dual graph as in Proposition 4.6 (3) with the weight of $B_{0}$ equal to 2 (by Corollary 7.4). Note that Remark 7.5 implies that such a fibration $\kappa$ and the curve $F_{0} \cup B_{0}$ can be constructed starting from a quadric.

The preimage of $F_{0} \cup B_{0}$ in $\bar{X}$ must be a curve with a dual graph $\Gamma^{\prime}$ such that $\Gamma$ is a subgraph of $\Gamma^{\prime}$ with contractible $\Gamma^{\prime} \ominus \Gamma_{0}$ and $C_{0} \in \Gamma_{0} \subset \Gamma$ being a linear vertex of $\Gamma$ with a nonnegative weight. Clearly if we can find such a graph $\Gamma^{\prime}$, a desired surface $\bar{X}$ together with a curve $G$ exist.

If $\Gamma^{\prime}=\Gamma_{0}$ then we have configuration (3) from Theorem 1.4 with $n=0$ and $w_{0}=2$. If $\Gamma=\Gamma_{0}$ and $\Gamma^{\prime} \ominus \Gamma$ consists of one (resp. two) (-1)-vertices then we have the same configuration with $n=0$ and $w_{0}=1$ (resp. $w_{0}=0$ ). In the case of $n \geq 1$ in configuration (3) $\Gamma \ominus \Gamma_{0}$ should be a linear chain $C_{1}+\ldots+C_{n}$ with weights $w_{i} \leq-2$. To show that $\Gamma^{\prime}$ containing such $\Gamma$ exists let $\Gamma^{\prime} \ominus \Gamma$ be a disjoint collection of $(-1)$-vertices such that $C_{i}$ has $-w_{i}-2$ neighbors from this collection for $1 \leq i \leq n-1$, while $C_{n}$ has $-w_{n}-1$ such neighbors. Then contraction of these $(-1)$-vertices transforms the chain into $[[-2,-2, \ldots,-2,-1]]$. Hence $\Gamma^{\prime} \ominus \Gamma_{0}$ is contractible and this contraction yields $w_{0}=1$. Joining, if necessary, $C_{0}$ with an extra $(-1)$-vertex from $\Gamma^{\prime} \ominus \Gamma$ one can make $w_{0}=0$ which yields all possibilities for configuration (3) listed in Theorem 1.4 $(\alpha)$. For configuration (5) $\Gamma \ominus \Gamma_{0}$ is of the form

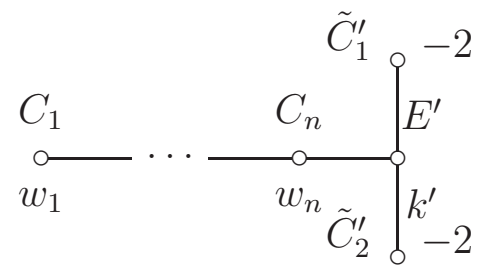

where $n \geq 0$ and $w_{i} \leq-2$ for $i \geq 1$. Consider $n \geq 1$. To get a contractible $\Gamma^{\prime} \ominus \Gamma_{0}$ one must have a contractible branch of $\Gamma^{\prime}$ at $E^{\prime}$ containing, say, $\tilde{C}_{1}^{\prime}$ (which takes place if the branch consists of $\tilde{C}_{1}^{\prime}$ and a $(-1)$-vertex). Contraction of this branch yields a linear chain in which the proper transforms of $E^{\prime}, \tilde{C}_{2}^{\prime}$ and $C_{n}$ will be denoted by $\breve{E}^{\prime}$, $\breve{C}_{2}^{\prime}$ and $\breve{C}_{n}$ respectively. Note that the weight of $\breve{E}^{\prime}$ is $k^{\prime}+1$. If $k^{\prime} \leq-3$ then we deal with a linear chain as in configuration (3) and the same argument produces a contractible $\Gamma^{\prime} \ominus \Gamma_{0}$ and $w_{0}=1$ or $w_{0}=0$. If $k^{\prime}=-2$ then $\breve{E}^{\prime}$ is a $(-1)$-vertex and we can contract $\breve{E}^{\prime}$ and $\breve{C}_{2}^{\prime}$. Note that this contraction changes the weight of $\breve{C}_{n}$ to $2+w_{n}$ and for further contraction we have to require that the latter number is negative, i.e. $w_{n} \leq-3$. This yields all configurations in Theorem 1.4 (5) with $n \geq 1$.

For $n=0$ consider the second fiber $F_{1}$ of $\kappa$ that meets $B_{0}$ at one point only. Make two monoidal transformations of $\hat{X}$ at $F_{1} \cap B_{0}$ and the infinitely near point. Then the graph of the preimage of $F_{0} \cup B_{0} \cup F_{1}$ yields configuration (5) with $n=0$ and $k^{\prime}=-1$. Using additional blowing up we can also make $k^{\prime} \leq-2$ which exhausts all configurations (5) listed in Theorem $1.4(\beta)$ and, thus, concludes the proof.

Proposition 7.7. Statement (2) of Proposition 7.1 is true.

Proof. Consider the cycle $((0,0,0,0))$ which is the dual graph of an SNC-curve in the quadric whose components are rational. This implies the part "if" of the statement.

Let $\Gamma=C_{0}+C_{1}+\ldots+C_{n}+C_{n+1}$ be a cyclic graph with $n \geq 2$, weights $w_{0}=w_{n+1}=0$ and $w_{i} \leq-2$ for $1 \leq i \leq n$ such that $\Gamma$ is the dual graph of an SNC curve in a 
smooth complete surface $\bar{X}$ with rational irreducible components. By Theorem $4.2 C_{0}$ generates a $\mathbb{P}^{1}$-fibration with $C_{1}$ and $C_{n+1}$ being sections of this fibration. Furthermore, $C_{2}+\ldots+C_{n}$ is contained in a fiber $F$ of the fibration. Note that the multiplicity of $C_{n}$ in $F$ is 1 since $C_{n}$ meets the section $C_{n+1}$. By Lemma $4.3 F$ is contractible to a zero vertex that is the proper transform of $C_{n}$. This contraction leads to a curve with a circular dual graph $C_{0}^{\prime}+C_{1}^{\prime}+C_{n}^{\prime}+C_{n+1}^{\prime}$ where $C_{i}^{\prime}$ is the proper transform of $C_{i}$ and the weights of $C_{0}^{\prime}, C_{n}^{\prime}$, and $C_{n+1}^{\prime}$ are zero. Note that $C_{1}^{\prime}$ is contained in a fiber of the fibration induced by $C_{n+1}^{\prime}$. Repeating the previous argument we can suppose that it coincides with this fiber, i.e its weight is zero which yields the desired conclusion.

Now Propositions 7.6 and 7.7 yield Proposition 7.1.

Example 7.8. (1) Let $n \geq 5$ and $k$ be the integer part of $n / 2$. Let $\Gamma_{0}=\left(\left(0,0, w_{1}, \ldots, w_{n}\right)\right)$ be a circular weighted graph with $w_{k}$ and $w_{k+1} \leq-2$ and $w_{i} \leq-3$ for $i \neq k, k+1$. Then $\Gamma_{0}$ is a dual graph of an SNC-curve with rational components contained in a smooth rational surface.

Indeed, consider $n-2$ consequent inner blowing up in the cycle $((0,0,0,0))$ so that for an even (resp. odd) $i$ the $i$-th blowing up occurs at the left (resp. right) edge adjoint to the vertex created in the previous step. Then we get the following cycle $((0,0,-3, \ldots,-3,-2,-1,-3, \ldots,-3))$ where -2 and -1 occupy positions $k$ and $k+1$ or vice versa. Now after appropriate outer blowing up this cycle can be transformed into the desired one.

(2) It is easy to show that the cycle $((0,0,-2,-2,-2,-2,-2))$ is not a subgraph of a graph contractible to $((0,0,0,0))$ and, thus, it cannot serve as the dual graph of an SNC-curve with rational components contained in a smooth surface.

\section{Proof of the necessity Part of the Main Theorem}

Setting 8.1. In this section we suppose that $\hat{X}$ is an SNC-completion of a smooth rational semi-affine surface $X$. As usual the dual graph of $\hat{D}=\hat{X} \backslash X$ will be denoted by $\Gamma$.

Definition 8.2. We say that an irreducible curve $F \subset X$ is distinguished if for any $\mathbb{C}$ - or $\mathbb{C}^{*}$-fibration $f: X \rightarrow \mathbb{C}$ this curve is contained in a singular fiber of $f$.

Any automorphism of $X$ that transforms fibers of $f$ into fibers of $f$ respects general fibers (since only in neighborhoods of general fibers the fibration is locally trivial). Hence, the following fact holds.

Lemma 8.3. Let $\nu$ be a complete vector field on $X$ that sends fibers of $f$ to fibers of $f$. Then the flow of $\nu$ preserves every singular fiber of $f$.

In combination with Theorem 1.5 this leads to the following important technical tool which has already appeared implicitly in Proposition 6.12.

Proposition 8.4. If a semi-affine surface $X$ contains a distinguished curve then its Remmert reduction $X_{0}$ is not generalized Gizatullin.

Proof. Let $C$ be a distinguished curve and let $\nu$ be a complete algebraic vector field on $X_{0}$. Then by Theorem 1.5 the flow of $\nu$ preserves a $\mathbb{C}$ - or $\mathbb{C}^{*}$-fibration $f$. Since $C$ by definition belongs to a singular fiber of $f$ it is preserved by Lemma 8.3. 
Setting 8.5. Recall that $\Gamma$ is contractible to a minimal graph $\Gamma_{\min }$ whose set of branch points $\operatorname{Br}\left(\Gamma_{\min }\right)$ is determined uniquely by Proposition 3.4. Consider the subset $S$ of $\operatorname{Br}\left(\Gamma_{\text {min }}\right)$ consisting of all branch points $E$ of valency 3 in $\Gamma_{\text {min }}$ such that

- two of branches at $E$ are just (-2)-vertices;

- by elementary transformations (as in Proposition 3.3) on connected components of $\Gamma_{\min } \ominus \operatorname{Br}\left(\Gamma_{\text {min }}\right)$ one can make the weight of $E$ equal to -1 .

Note that $S$ is also determined uniquely by Proposition 3.4 and Corollary 3.5. Let $T_{\min }=\operatorname{Br}\left(\Gamma_{\min }\right) \backslash S$ and $\Gamma_{0, \min }, \Gamma_{1, \min }, \ldots, \Gamma_{n, \min }$ be connected components of $\Gamma_{\min } \ominus T_{\min }$. Let $T$ be the set of the branch points in $\Gamma$ corresponding to $T_{\min }$ and $D_{0}, D_{1}, \ldots, D_{n} \subset$ $\hat{D}$ be the curves in associated with the connected components of $\Gamma \ominus T$.

Connectedness of $\hat{D}$ and the description of vertices from $T_{0}$ in Setting 8.5 imply the following.

Lemma 8.6. Each graph $\Gamma_{i, \min }$ has one of the following configurations:

(a) linear graph;

(b) circular graph;

$(c)$

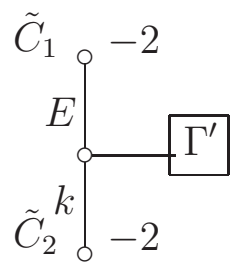

$(d)$

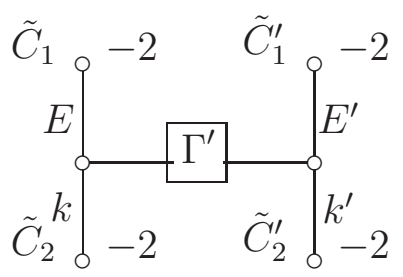

where $\Gamma^{\prime}$ is a linear graph or empty. Furthermore,

- in (b) and (d) one has $\Gamma_{i, \min }=\Gamma_{\min }$;

- in (a) there are at most two neighbors of $\Gamma_{i, \min }$ in $\Gamma_{\min }$, each of them is contained in $T_{1, \min }$ and joined by an edge with an end-vertex of $\Gamma_{i, \min }$;

- in (c) there is at most one neighbor of $\Gamma_{i, \min }$ in $\Gamma_{\min }$, it is contained in $T_{1, \text { min }}$ and joined by an edge with the right end-vertex of $\Gamma^{\prime}$.

Lemma 8.7. Let $\hat{f}: \hat{X} \rightarrow \mathbb{P}^{1}$ be a proper extension to $\hat{X}$ of a $\mathbb{C}$ - or $\mathbb{C}^{*}$-fibration $f: X \rightarrow \mathbb{C}$, i.e., some fiber $F$ of $\hat{f}$ has a support in $\hat{D}$. Then this support is, in fact, contained in some $D_{i}\left(\right.$ say, $\left.D_{0}\right)$. 
Proof. Making contractions in $\hat{D}$ we get a pseudo-minimal extension $\hat{g}: \hat{X}^{\prime} \rightarrow \mathbb{P}^{1}$ of $f$ with dual graph $\Gamma^{\prime}$ for $\hat{D}^{\prime}=\hat{X}^{\prime} \backslash X$. By Proposition $4.6 \Gamma^{\prime}$ contains either a linear 0 -vertex $C$ or a subgraph of type $\Gamma_{*}$ which includes a chain $[[-2,-1,-2]]$ consisting of vertices $C_{1}+E+C_{2}$.

Since the image of $C$ in $\Gamma_{\min }$ is a linear vertex it does not belong to $T_{\min }$, i.e., the preimage of $C$ in $\hat{D}$ (which is a support of a fiber of $\hat{f}$ ) is contained in $D_{i}$.

In the case of the chain $C_{1}+E+C_{2}$ the images of $C_{1}$ and $C_{2}$ in $\Gamma_{\text {min }}$ are linear vertices, while the image of $E$ is either a linear vertex of a nonnegative weight or a branch point of valency 3 which does not belong to $T_{\min }$. Hence, the preimage of the chain in $\hat{D}$ is contained in some $D_{i}$ which is the desired conclusion.

Proposition 8.8. Let $\hat{f}: \hat{X} \rightarrow \mathbb{P}^{1}$ and $\hat{g}: \hat{X} \rightarrow \mathbb{P}^{1}$ be proper extensions to $\hat{X}$ of $\mathbb{C}$ or $\mathbb{C}^{*}$-fibrations on $X$. Suppose that there is a fiber $F$ of $\hat{f}$ and a fiber $G$ of $\hat{g}$ with supports in different $D_{i}$ 's. Then $\hat{f}$ and $\hat{g}$ coincide (up to an automorphism of $\mathbb{P}^{1}$ ) and, furthermore, every $\mathbb{P}^{1}$-fibration $\hat{h}: \hat{X} \rightarrow \mathbb{P}^{1}$ which extends a $\mathbb{C}$-or $\mathbb{C}^{*}$-fibration on $X$ coincides with $\hat{f}$.

Proof. By assumption $F \cdot G=F^{2}=G^{2}=0$ and for every section $S$ of $\hat{f}$ one has $F \cdot S=1$. Hence, by the Hodge index theorem $F$ and $G$ generate proportional elements $[F]$ and $[G]$ of $H^{2}(\hat{X}, \mathbb{Z})$, i.e., $n[F]=m[Q]$ for some nonzero $n, m \in \mathbb{Z}$. Note that $m=n$ since otherwise, say in the case of $m>n$, the product $G \cdot S$ is either negative or not an integer number. Thus, these divisors $F$ and $G$ are equivalent and $\mathbb{P}^{1}$-fibrations $\hat{f}$ and $\hat{g}$ (generated by the linear systems of $F$ and $G$ ) coincide (up to an automorphism of the image $\mathbb{P}^{1}$ ). By Lemma $8.7 \hat{h}$ has a fiber with support in some $D_{j}$. Note that $D_{j}$ does not contain the support of either $F$ or $G$. Hence, $\hat{h}$ coincides with either $\hat{f}$ or $\hat{g}$ and we are done.

Corollary 8.9. Suppose that $\hat{X}$ admits two distinct $\mathbb{P}^{1}$-fibrations $\hat{f}: \hat{X} \rightarrow \mathbb{P}^{1}$ and $\hat{g}: \hat{X} \rightarrow \mathbb{P}^{1}$ that extend $\mathbb{C}$ - or $\mathbb{C}^{*}$-fibrations on $X$. Then both of them have fibers with support in the same $D_{i}\left(\right.$ say, $\left.D_{0}\right)$ and no fibers with support in any $D_{j}$ where $j \neq i$.

Proposition 8.10. If $X$ is generalized Gizatullin then distinct fibrations $\hat{f}$ and $\hat{g}$ as in Corollary 8.9 exist. In particular, $\Gamma_{0, \min }$ cannot be a 0 -vertex.

Proof. Assume that there is only one $\mathbb{P}^{1}$-fibration $\hat{f}$ of this type. Then its restriction $f: X \rightarrow B:=f(X) \subset \mathbb{C}$ has no singular fibers by Proposition 8.4 and Lemma 8.3, i.e., $f$ is a locally trivial $\mathbb{C}$ - or $\mathbb{C}^{*}$-fibration over $B$. Furthermore, $B$ is not hyperbolic since otherwise for every complete vector field $\nu$ the image of any integral curve under $f$ must be constant, i.e., $\nu$ must be tangent to the fibers of $f$ and $X$ has no open orbit. That is, $B$ is either $\mathbb{C}$ or $\mathbb{C}^{*}$. This implies that $X$ is isomorphic to either $\mathbb{C}^{2}$, $\mathbb{C} \times \mathbb{C}^{*},\left(\mathbb{C}^{*}\right)^{2}$ or a twisted locally trivial $\mathbb{C}^{*}$-fibration over $\mathbb{C}^{*}$. In the first three cases our assumption is obviously wrong.

In the last case $\hat{D}$ contains two fibers of $\hat{f}$ whose graphs under pseudo-minimality assumption are the chains $[[-2,-1,-2]]$ by Proposition 4.6. That is, $\Gamma$ is of the form (d) in Lemma 8.6 with $\Gamma^{\prime}$ being the component $C_{0}$ of $\hat{D}$ that is ramified double cover of $\mathbb{P}^{1}$ under $\hat{f}$ and $k=k^{\prime}=-1$. In order to avoid the existence of another fibration $\hat{g}$ 
one needs to require that $C_{0}^{2} \leq-1$. However, in this case contracting the chains we get a Hirzebruch surface with the image of $C_{0}$ having self intersection at most 3 contrary to Proposition 7.3 which concludes the proof.

Example 8.11. The last surface $S$ which is a twisted locally trivial $\mathbb{C}^{*}$-fibration over $\mathbb{C}^{*}$ is rather interesting. Given a coordinate $z$ on a fiber $\mathbb{C}^{*}$ one can suppose that the monodromy around the puncture in the base in given by $z \rightarrow 1 / z$. Treating $\mathbb{C}^{*}$ as a complexification of a circle both in the base in the fiber one can see that $S$ is nothing but the complexification of the Klein bottle. An SNC-completion of $S$ can be constructed in the following way. Consider the parabola $C$ given by $x-y^{2}=0$ in $\mathbb{P}_{x}^{1} \times \mathbb{P}_{y}^{1}$. Blow up point $(0,0)$ (resp. $(\infty, \infty)$ ) and an infinitely near point. The resulting surface $\hat{S}$ is the desired completion of $S$ with fibers over $x=0$ and $x=\infty$ being $[[-2,-1,-2]]$-chains in $\hat{S} \backslash S$ and the proper transform $C_{0}$ of $C$ playing the role of the ramified double cover with $C_{0}^{2}=0$. There are only two $\mathbb{C}^{*}$-fibrations $x$ and $y^{2} / x$ on $S$ corresponding to the $\mathbb{P}^{1}$-fibrations associated with the chains $[[-2,-1,-2]]$ and vertex $C_{0}$ respectively. Both $x$ and $y^{2} / x$ are rational first integrals (the first one for the complete algebraic field $\nu_{1}=\left(y^{2}-x\right) \partial / \partial y$ and the second one for $\left.\nu_{2}=2 x \partial / \partial x+y \partial / \partial y\right)$. There is also a complete algebraic field $\nu=\nu_{1}+\nu_{2}{ }^{11}$ for which neither $x$ nor $y^{2} / x$ is a rational first integral (and which, therefore, has no rational first integral at all). Note that the function $x$ yields the preserved fibration on $S$.

Remark 8.12. Proposition 8.10 does not hold in general for surfaces with open orbits. Indeed, consider surfaces $y x^{2}+y^{n}+z^{2}=0, n \geq 4$ with du Val singularities of type $D_{n}$ mentioned in Example 6.13. Each of them (say $S$ ) has a complete algebraic field tangent to the $\mathbb{C}^{*}$-fibration described in that example and the semi-simple field associated with the $\mathbb{C}^{*}$-action $(x, y, z) \rightarrow\left(\lambda^{n-1} x, \lambda^{2} y, \lambda^{n} z\right)$. Hence, it has an open $\operatorname{AAut}_{\text {hol }}(S)$-orbit. On the other hand there is an SNC-completion of $S$ with dual graph of the divisor at infinity as in Proposition 6.10 whose form in combination with Proposition 4.6 yields a unique $\mathbb{C}^{*}$-fibration and the absence of $\mathbb{C}$-fibrations on $S$.

Proposition 8.13. In the Settings 8.1 and 8.5 let the Remmert reduction $X_{0}$ of $X$ be a generalized Gizatullin surface. Then $T$ is empty and in particular $\Gamma_{\min }$ has one of the four configurations from Lemma 8.6.

Proof. By Lemma 8.6 it suffices to consider configurations (a) and (c) and show that $T$ is empty.

Configuration (a). Assume that $\Gamma_{\min } \neq \Gamma_{0 \text {,min }}$, i.e., there is a vertex $V \in T_{\min }$ adjacent to the right end $E_{1}$ of $\Gamma_{0, \text { min }}$. By Proposition 3.3 we can suppose that the left end $E_{0}$ of $\Gamma_{0, \text { min }}$ is a 0 -vertex and by Proposition $8.10 E_{0} \neq E_{1}$.

That is, the $\mathbb{P}^{1}$-fibration $\hat{f}: \hat{X} \rightarrow \mathbb{P}^{1}$ induced by $E_{0}$ is an an extension

(i) of a $\mathbb{C}$-fibration if $E_{0}$ has no neighbor from $T_{\min }$; or

(ii) of a $\mathbb{C}^{*}$-fibration if $E_{0}$ has such a neighbor $W$ (where we allow equality $W=V$ ).

In (i) (and even in (ii) when $W \neq V$ ) $V$ is not a section of the fibration $\hat{f}$ and is contained in a fiber $\hat{f}^{-1}(a)$ of $\hat{f}$. By Lemma $4.8 f^{-1}(a)$ is a singular fiber of $f$ that contains $F=C \cap X \simeq \mathbb{C}$ where $C$ is a component of $\hat{f}^{-1}(a)$ that does not meet $D_{0}$.

\footnotetext{
${ }^{11}$ Completeness of $\nu$ can be extracted from completeness of $\nu_{1}$ and $\nu_{2}$ and the fact that $\left[\nu_{1}, \nu_{2}\right]=$ $-\nu_{1}$.
} 
Given a different $\mathbb{C}$ - or $\mathbb{C}^{*}$-fibration $g: X \rightarrow \mathbb{C}$ by the Zariski theorem we can find an SNC-completion $\bar{X}=X \cup \bar{D}$ of $X$ which dominates $\hat{X}$ and admits a proper extension $\bar{g}$ of $g$. The preimage $\bar{D}_{0}$ of $D_{0}$ in $\bar{D}$ has dual graph $\bar{\Gamma}_{0}$ which contains the dual graph $G_{0}$ of the preimage of $E_{0}$ and by Proposition 8.8 the dual graph $G$ of the support of a fiber of $\bar{g}$. Note that the proper transform $\bar{C}$ of $C$ is again contained in the fiber of $\bar{g}$ because it does not meet $\bar{D}_{0}$. By the Zariski lemma (e.g., see [4, p. 90]) $G_{0} \backslash G$ cannot be empty. This implies that either the proper transform of $V$ is not a section of the $\mathbb{P}^{1}$-fibration $\bar{g}$ or there are two neighbors of $G$ in $\bar{D}$ which means that $g$ is a $\mathbb{C}^{*}$-fibration on $X$. In both cases $F \simeq \mathbb{C}$ is a component of a singular fiber of $g$ and, thus, it is a distinguished curve. Since it survives the Remmert reduction we see that $X_{0}$ is not generalized Gizatullin by Proposition 8.4. A contradiction, i.e., $T$ must be empty in this case.

When $W=V$ we deal only with $\mathbb{C}^{*}$-fibrations. Blowing up the edge between $E_{1}$ and $V$, if necessary, replace $\Gamma_{0, \text { min }}$ with a linear graph $\Gamma_{0}$ which contains at least three vertices. Using reconstructions as in Proposition 3.3 we can change $\Gamma_{0}$ so that it contains now a 0-vertex $E$ somewhere in the middle (i.e., $E$ is not a neighbor of $V$ ). Lemma 4.8 supplies us as before with a distinguished curve $F \simeq \mathbb{C}$ which concludes the consideration of configuration (a).

Configuration (c). Consider $\hat{f}: \hat{X} \rightarrow \mathbb{P}^{1}$ induced by the chain $\tilde{C}_{1}+E+\tilde{C}_{2}=$ $[[-2,-1,-2]]$ contained in $D_{0}$. Note that $D_{0}$ contains an irreducible component $U$ meeting $E$ transversely. Since the multiplicity of $E$ in the fiber of $\hat{f}$ is two the map $\left.\hat{f}\right|_{U}: U \rightarrow \mathbb{P}^{1}$ has degree two. This implies that $f$ is a $\mathbb{C}^{*}$-fibration on $X$. If there exists $V \in T$ then $V \neq U$ since otherwise the second fibration $\hat{g}$ does not exists. Hence, by Lemma 4.8 we have again a singular fiber of $f$ containing an irreducible component $F$ isomorphic to $\mathbb{C}$ and the same argument shows that $g$ as before is also a $\mathbb{C}^{*}$-fibration with $F$ being a component of a fiber of $g$. This yields a distinguished curve and the desired conclusion.

Now we are able to prove one direction of the Theorem 1.4, namely the necessary condition for a semi-affine surface to be generalized Gizatullin.

Proposition 8.14. Let $X$ be a semi-affine surface which is generalized Gizatullin, then $X$ possesses a completion $\bar{X}$ where the dual graph of $\bar{X} \backslash X$ is of one of the forms (1) - (6) from Theorem 1.4.

Proof. We go through the cases of Lemma 8.6.

For the case of a linear dual graph the Proposition is well known. Let us consider first circular graphs. By [11, Proposition 3.28] they can be reduced via birational transformations to the following standard (and "essentially unique") forms:

(i) $\left(\left(0_{2 k}, w_{1}, \ldots, w_{n}\right)\right)$ with $k \geq 0, n>0$, and $w_{i} \leq-2$; or

(ii) $\left(\left(0_{l}, w\right)\right)$ with $l>0$ and $w \leq 0$; or

(iii) $\left.\left(0_{2 k},-1,-1\right)\right)$ with $k \geq 0$

where the subindex reflects the number of consequent zero weights. Note that in (i) we disregard the case of $k=0$ (since the intersection matrix of such a graph is negative definite contrary to the Nakai-Moishezon criterion) and $k \geq 2$ (to avoid a contradiction with the Hodge index theorem). Similarly, in (ii) we omit the case of 
$l=1$ and $w \leq-1$ because otherwise we get only one $\mathbb{P}^{1}$-fibration as in Proposition 8.9 contrary to Proposition 8.10. The Hodge index theorem implies also that in (ii) $l \leq 3$. Hence, the remaining possibilities in (i) and (ii) produce (2a) and (2b). By the same arguments in (ii) we have to consider only weights as in $(2 \mathrm{c})$.

By Proposition 8.13 it suffices now to consider $\Gamma_{\min }$ as in configurations (c) and (d) in Lemma 8.6 and show that $\Gamma^{\prime}$ is of the desired form.

Consider first the case when there exists a non negative vertex in a minimal graph of $\Gamma^{\prime}$. After blowing up which keeps the graph linear we can always suppose that it is actually of weight 0 . Using operations of of form $[[v, 0, w]] \rightarrow[[v-1,0, w+1]]$ as in Proposition 3.3 we can suppose furthermore, that this vertex is the left endpoint $C_{0}$ of $\Gamma^{\prime}$ and the weight $k$ in configurations (c) and (d) from Lemma 8.6 is -1 . Proposition 7.1 implies now that $\Gamma$ must be of desired form which describes configurations (3) and (5) in Theorem 1.4 completely.

Now consider the case when there is no non negative vertex in a minimal graph of $\Gamma^{\prime}$. If in (d) $\Gamma^{\prime}$ is not empty then the divisor

$$
C_{1}+\ldots+C_{n}+\tilde{C}_{1}^{\prime}+E^{\prime}+\tilde{C}_{2}^{\prime}
$$

is contained in the same fiber of $\hat{f}$. Hence, by the Zariski lemma (e.g., see [4, p. 90]) we have not only $C_{i}^{2} \leq-2$ but also $\left(E^{\prime}\right)^{2} \leq-1$ since the intersection matrix of this divisor must be negative definite. By Corollary 3.5 we see that this minimal graph is unique and $\hat{f}$ is the only extension of a $\mathbb{C}$ - or $\mathbb{C}^{*}$-fibration on $X$ contrary to Proposition 8.10. Thus, $\Gamma^{\prime}$ is empty in this case and we have (6) (condition $k^{\prime} \geq-1$ is necessary to provide a second $\mathbb{P}^{1}$-fibration not equal to $\hat{f}$ ).

In the absence of a non negative vertex for (c) we have $k=-1$ and unless $w_{0}=-2$ there is again the same contradiction with Proposition 8.10. Thus, $w_{0}=-2$ and we have three $\mathbb{P}^{1}$-fibrations associated with with the chains $\tilde{C}_{1}+E+\tilde{C}_{2}, \tilde{C}_{1}+E+C_{0}$, and $\tilde{C}_{2}+E+C_{0}$ respectively. However, if $n \geq 1$ the restriction of any of the last two fibrations to $X$ is not a $\mathbb{C}^{*}$-fibration because contrary to Proposition $4.6(3 \mathrm{~b})$ the $\tilde{C}_{i}+E+C_{0}$ meets not only the ramified double cover but also $C_{1}$. This leads to case (4) and we are done.

\section{Proof of the Sufficiency part of the Main Theorem}

Lemma 9.1. Let $X$ be a smooth semi-affine surface. Suppose that $f_{i}: X \rightarrow \mathbb{C}, i=1,2$ are either $\mathbb{C}$ or $\mathbb{C}^{*}$-fibrations such that the intersection of every pair of non-singular fibers of $f_{1}$ and $f_{2}$ is a finite non-empty set. Let $S_{i}$ be the union of singular fibers of $f_{i}$. Then there is an open orbit $U$ of the natural $\mathrm{AAut}_{\mathrm{hol}}(X)$-action (Definition 1.3) on $X$ such that its complement is contained in $S_{1} \cap S_{2}$.

Proof. Let $x \notin S_{1} \cap S_{2}$ and let $U$ be the orbit of $x$. Say $x$ is contained in a non-singular fiber $E_{1}$ of $f_{1}$. By Lemma $6.5 E_{1} \subset U$, i.e., we can suppose that $x$ is an arbitrary point of $E_{1}$. In particular, we can suppose that $x \in E_{1} \cap E_{2}$ where $E_{2}$ is a given non-singular fiber of $f_{2}$. By Lemma $6.5 E_{2} \subset U$. Similarly any given fiber of $f_{1}$ is contained in $U$ and we are done. 
Proof of Theorem 1.4. By Proposition 8.14 it suffices to show that every normal affine algebraic surface $X_{0}$ with a dual graph of the boundary appearing in Theorem 1.4 is a generalized Gizatullin surface.

If $X_{0}$ is a Gizatullin surface then it is known that $X_{0}$ is already quasi-homogeneous under the algebraic automorphisms by [16]. Thus, we need to show now that cases (2)-(6) in Theorem 1.4 indeed present surfaces quasi-homogeneous under the group of algebraically generated automorphisms. Suppose that $X \rightarrow X_{0}$ is a minimal resolution of singularities, i.e., $X$ is smooth semi-affine. Consider the most difficult dual graph $\Gamma$ of $\bar{D}=\bar{X} \backslash X$ as in Figure (5). Making inner blowing up if necessary we can suppose that the weight of $C_{0}$ is zero. Let $f: X \rightarrow \mathbb{C}$ be the twisted $\mathbb{C}^{*}$-fibration associated with the subgraph $K=\tilde{C}_{1}+E+\tilde{C}_{2}$ and $f_{0}: X \rightarrow \mathbb{C}$ be the untwisted $\mathbb{C}^{*}$-fibration associated with the 0 -vertex $C_{0}$. Suppose that $\bar{f}: \bar{X} \rightarrow \mathbb{P}^{1}$ and $\bar{f}_{0}: \bar{X} \rightarrow \mathbb{P}^{1}$ are their proper extensions. Let $S$ (resp $S_{0}$ ) be the union of singular fibers of $\bar{f}$ (resp. $\bar{f}_{0}$ ) that meet $C_{0}$ (resp. $K$ ) and $S^{\prime}$ (resp. $S_{0}^{\prime}$ ) be the union of such fibers that do not. That is, each fiber from $S^{\prime}$ (resp. $S_{0}^{\prime}$ ) meets one of curves $C_{1}, \ldots, C_{n}, E^{\prime}, \tilde{C}_{1}^{\prime}, \tilde{C}_{2}^{\prime}$. Let $U$ be the open orbit of the natural $\mathrm{AAut}_{\text {hol }}(X)$-action in $X$. By Lemma 9.1 $X \backslash U$ is contained in $\left(S \cup S^{\prime}\right) \cap\left(S_{0} \cup S_{0}^{\prime}\right)$. On the other hand, since $S$ is not contained in a fiber of $\bar{f}_{0}$, with an exception of a finite set $S$ is contained in $U$ by Lemma 6.5. The same is true for $S_{0}$. Thus, $X \backslash U$ is contained in $\left(S^{\prime} \cap S_{0}^{\prime}\right) \cup T_{0}$ where $T_{0}$ is a finite set. That is, we need to show that up to a finite set every curve $F \subset S^{\prime} \cup S_{0}^{\prime}$ that is a component of singular fibers of both $\bar{f}$ and $\bar{f}_{0}$ is contained in $U$. Let $F$ meet $C_{1}$. Then by Proposition 3.3 after a sequence of elementary transformations such that all vertices of $\Gamma$ but $C_{0}$ survive we can make the weight of $C_{1}$ equal to 0 . In particular, this 0 -vertex yields a $\mathbb{C}^{*}$-fibration $f_{1}: X \rightarrow \mathbb{C}$. Since $F$ meets $C_{1}$ we see that $F \cap X$ is not contained in a fiber of $f_{1}$ and, therefore, by Lemma 6.5 one has $F \backslash T_{1} \subset U$ where $T_{1}$ is a finite set.

Similarly, by Proposition 3.3 if $i \geq 2$ then after a sequence of elementary transformations under which all vertices $C_{i}, \ldots, C_{n}, E^{\prime}, \tilde{C}_{1}^{\prime}, \tilde{C}_{2}^{\prime}$ survive we can make the weight of $C_{i}$ equal to 0 . Thus, the same argument implies that if $F$ meets $C_{i}$ then $F \backslash T_{i} \subset U$ where $T_{i}$ is a finite set. In the case of $F$ meeting of the curves $E^{\prime}, \tilde{C}_{1}^{\prime}$, or $\tilde{C}_{2}^{\prime}$ using elementary transformations in the chain $C_{0}+\ldots+C_{n}$ we can make the weight of $E^{\prime}$ equal to -1 . Then $\tilde{C}_{1}^{\prime}+E^{\prime}+\tilde{C}_{2}^{\prime}$ becomes a subgraph that induces a twisted $\mathbb{C}^{*}$-fibration whose restriction to $F \cap X$ is not constant. That is, up to a finite set $F$ is contained in $U$. Hence, we are done in case (5).

The argument for case (3) and a circular graph in case (2) are similar (say, the only difference in (3) is that when one makes the weight of $C_{n}$ equal to 0 then the associated fibration is a $\mathbb{C}$-fibration and not a $\mathbb{C}^{*}$-fibration).

Also in case (4) the argument is similar, we have to work with the three twisted $\mathbb{C}^{*}$-fibrations associated with the three $[[-2,-1,-2]]$ subgraphs.

If $k^{\prime} \geq 0$ in case (6) then one needs to make a sequence of inner blow-ups over the edge between $E$ and $E^{\prime}$ such that the resulting graph looks like

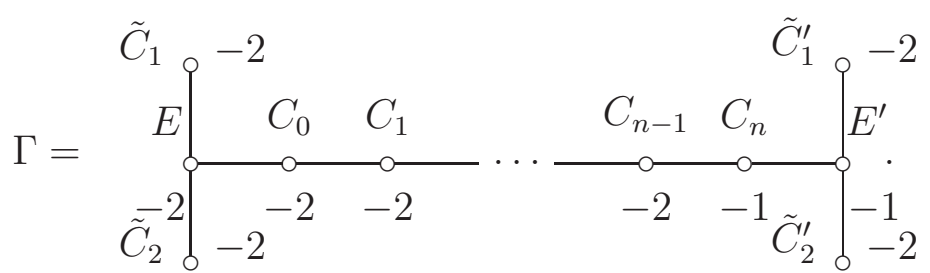


Then we have two twisted $\mathbb{C}^{*}$-fibrations $g^{\prime}: X \rightarrow \mathbb{C}$ and $g: X \rightarrow \mathbb{C}$ induced by the subgraphs $K^{\prime}=\tilde{C}_{1}^{\prime}+E^{\prime}+\tilde{C}_{2}^{\prime}$ and $K=\Gamma \ominus K^{\prime}$, indeed $K$ is contractible to a $[[-2,-1,-2]]$ subgraph by contracting $C_{0}+\ldots+C_{n}$. Suppose that $\bar{X}$ is the SNCcompletion of $X$ with the boundary described by the graph above and $\bar{g}: \bar{X} \rightarrow \mathbb{P}^{1}$ (resp. $\bar{g}^{\prime}: \bar{X} \rightarrow \mathbb{P}^{1}$ ) is a proper extension of $g\left(\right.$ resp. $g^{\prime}$ ). Note that the singular fibers of $\bar{g}$ must meet $K^{\prime}$ but not $K$ while for the singular fibers of $\bar{g}^{\prime}$ the situation is reversed. In particular, only complete curves that are contained in $X$ may be common components of singular fibers of $\bar{g}$ and $\bar{g}^{\prime}$. By Lemma $9.1 U$ is contained in the complement to the union of such components in $X$. Hence, $X_{0}$ is a generalized Gizatullin surface since these components are contractible to points in the Remmert reduction.

In the case of $k^{\prime}=-1$ a similar argument works and we are done.

\section{Homogeneity}

Setting 10.1. In this section $X$ is a smooth affine surface with an SNC-completion $\bar{X}$ such that the dual graph of $\bar{D}=\bar{X} \backslash X$ is one of those in Theorem 1.4. In particular, $X$ is a generalized Gizatullin surface.

Note that if $X$ admits a $\mathbb{C}$ or $\mathbb{C}^{*}$-fibration without singular fibers (say, $X$ is the complexification of the Klein bottle from Example 8.11) then it is homogeneous with respect to the $\mathrm{AAut}_{\text {hol }}(X)$-action because of the absence of fixed points for this action by virtue of Lemma 6.5. The same remains true in several other cases.

Theorem 10.2. Let $\bar{D}$ have a circular dual graph as in (2) of Theorem 1.4. Then $X$ is homogeneous with respect to the natural $\mathrm{AAut}_{\mathrm{hol}}(X)$-action.

Proof. Suppose that $C_{0}, \ldots, C_{n}$ are irreducible components of $\bar{D}$ such that $C_{0}^{2}=C_{1}^{2}=0$ and $C_{i}^{1} \leq-1$ for $i \geq 2, C_{i} \cdot C_{j}=1$ for $|i-j|=1$ or $\{i, j\}=\{0, n\}$, and $C_{i} \cdot C_{j}=0$ otherwise. Let $\bar{f}: \bar{X} \rightarrow \mathbb{P}^{1}$ be the $\mathbb{P}^{1}$-fibration associated with $C_{0}$, the $\mathbb{C}^{*}$-fibration $f$ be its restriction to $X$ and let $\left\{F_{i}\right\}$ be the irreducible components of singular fibers of $f$.

Case 1: $n \geq 3$. The absence of branch points in the dual graph of $\bar{D}$ and the smoothness of $X$ imply that all singular fibers of $\bar{f}$ but the one containing $C_{2}$ (say $\left.\bar{f}^{-1}(0)\right)$ are chains $[[-1,-1]]$ while $\bar{f}^{-1}(0)$ consists of the chain $\mathcal{C}=C_{2}+\ldots+C_{n-1}$ (joining sections $C_{1}$ and $C_{n}$ ) and some other components adjacent to smooth points of this chain which are $(-1)$-curves because of Lemma 4.3. Each of these $(-1)$-curves is of course a closure $\bar{F}_{i}$ of some $F_{i}$ and it is a component of a singular fiber of $\mathbb{P}^{1}$-fibration associated with $C_{1}$.

Hence, by Lemma 9.1 one can suppose that a potential fixed point of the $\operatorname{AAut}_{\text {hol }}(X)$ action is contained not only in a chain $[[-1,-1]]$ mentioned before but also in some $F_{i}$ from $f^{-1}(0)$. Therefore, it is enough to show that for any given $F_{i}$ there is a complete algebraic vector field whose restriction to $F_{i}$ is locally nilpotent and nontrivial, i.e., it generates a translation on $F_{i}$. Though a priori $F_{i}$ may be adjacent to any $C_{j}$ with $2 \leq j \leq n$ a reconstruction as in Proposition 3.3 enables us to consider only the case when $F_{i}$ is adjacent to $C_{2}$.

Contracting in fibers of $\bar{f}$ irreducible component not adjacent to $C_{1}$ (in particular $C_{2}$ is not contracted) we get a morphism $\varphi: \bar{X} \rightarrow \bar{X}^{\prime}$ into a Hirzebruch surface $\bar{X}^{\prime}$ with 
$C_{1}^{\prime}$ and $C_{n}^{\prime}$ playing the roles of disjoint sections where $C_{i}^{\prime}$ is the proper transform of $C_{i}$ in $\bar{X}^{\prime}$. That is, $\bar{X}^{\prime} \backslash C_{0}^{\prime}$ is naturally isomorphic to $\mathbb{C}_{x} \times \mathbb{P}_{y}^{1}$ with $C_{1}^{\prime} \backslash C_{0}^{\prime}, C_{2}^{\prime} \backslash C_{0}^{\prime}, C_{n}^{\prime}$ given by $\{y=\infty\},\{x=0\},\{y=0\}$.

Lemma 6.1 implies now that the pull-back of the vector field $\mu=y \frac{\partial}{\partial y}$ on $\bar{X}^{\prime} \backslash C_{0}^{\prime}$ is a rational vector field $\bar{\mu}$ on $\bar{X} \backslash C_{0}$ which has only simple poles and they are located on those $\bar{F}_{i}$ 's that are adjacent to the chain $\mathcal{C}$. This means that $x \mu$ induces a regular vector field $\nu$ on $X$ and even on $\bar{X} \backslash C_{0}$.

Note that $\varphi\left(F_{i}\right)=\left(0, y_{0}\right) \in \mathbb{C}^{*} \times \mathbb{P}^{1}$ with $y_{0} \neq 0, \infty$ and $F_{i}$ is obtained as the result of a monoidal transformation at this point. That is, one can introduce a local coordinate system $(u, v)$ on $X$ such that $\varphi(u, v)=\left(u, u v+y_{0}\right)$ and $F$ is given by equation $u=0$. Then $\nu$ is given by $\left(u v+y_{0}\right) \frac{\partial}{\partial v}$, i.e., its restriction to $F_{i}$ is nonzero and locally nilpotent. Hence, no point of $F_{i}$ is fixed under the $\operatorname{AAuthol}_{\text {hol }}(X)$-action which implies the desired conclusion in this case.

Case 2: $n=2$. One can blow up the edge between $C_{1}$ and $C_{2}$ to get an extra vertex $C$, i.e., we have four vertices in the new dual graph. Consider $\bar{f}, f, F_{i}$, and $\bar{f}^{-1}(0)$ as before. Note that the weight of the proper transform of $C_{1}$ becomes -1 but elementary transformation from Proposition 3.3 can make it again zero while keeping $C$ intact. That is, any $F_{i}$ from $\bar{f}^{-1}(0)$ is contained in a singular fiber of a $\mathbb{C}^{*}$-fibration on $X$ different from $f$. Lemma 9.1 implies that it suffices again to construct a translation on $F_{i}$ and the previous argument works.

Case 3: $n=1$ and $\bar{D}$ consisting of two 0-components $C_{0}$ and $C_{1}$ meeting each other transversely at two points. It requires a different approach which we sketch below. Let $\bar{f}$ be again the $\mathbb{P}^{1}$-fibation on $\bar{X}$ associated with $C_{0}$. Making contraction $\varphi: \bar{X} \rightarrow \bar{X}^{\prime}$ in the fibers of $\bar{f}$ we get a Hirzebruch surface $\bar{X}^{\prime}$ with the proper transform $C_{1}^{\prime}$ of $C_{1}$ playing the role of a ramified double section. Let $\bar{g}: \bar{X}^{\prime} \rightarrow \mathbb{P}_{x}^{1}$ be induced by $\bar{f}$. Without loss of generality we can suppose that $C_{0}^{\prime}=\bar{g}^{-1}(1)$ while 0 and $\infty$ are the only singular values of $\left.\bar{g}\right|_{C_{1}^{\prime}}$ as in Remark 4.5. Furthermore, applying the same reconstruction we exploited in Proposition 8.10 we can suppose that $C_{1}^{\prime}$ is the closure of the curve given by $x=y^{2}$ in $\bar{X}^{\prime} \backslash \bar{g}^{-1}(\infty) \simeq \mathbb{C}_{x} \times \mathbb{P}_{y}^{1}$. That is, the restriction $g$ of $\bar{g}$ to $X^{\prime}=\bar{X}^{\prime} \backslash\left(C_{0}^{\prime} \cup C_{1}^{\prime}\right)$ is a $\mathbb{C}^{*}$-fibration with two singular fibers $g^{-1}(0)$ and $g^{-1}(\infty)$ (both isomorphic to $\mathbb{C}$ ).

Since $X$ does not contain complete curves the surface $\bar{X}$ is obtained from $\bar{X}^{\prime}$ by several monoidal transformations at different points of $C_{1}^{\prime}$. Hence, the singular fibers of $f$ are $f^{-1}(0), f^{-1}(\infty)$ (each of them consists of one or two connected components isomorphic to $\mathbb{C}$ ) and fibers that are unions of form $F_{1} \cup F_{2}$ where $F_{i} \simeq \mathbb{C}$ and $F_{1}$ meets $F_{2}$ transversely at one point.

The vector field $\frac{\left(x-y^{2}\right)}{x-1} \frac{\partial}{\partial y}$ is regular and complete on $X^{\prime}$ and its restriction to $g^{-1}(0)$ and $g^{-1}(\infty)$ induces nontrivial translations. Furthermore, a calculation shows that it induces a regular vector field $\nu$ on $X$ which is a translation on every irreducible component of $f^{-1}(0)$ or $f^{-1}(\infty)$. Therefore, by Lemma 6.1 points of type $F_{1} \cap F_{2}$ are the only potential fixed points of the $\operatorname{AAuthol}_{\text {hol }}(X)$-action.

Consider the following reconstruction of the boundary divisor: Blow up one of edges between $C_{0}$ and $C_{1}$ and contract the proper transform of $C_{1}$. The resulting completion $\hat{X}$ of $X$ has the boundary divisor $\hat{D}$ consisting of two 0 -vertices $\hat{C}_{0}$ and $\hat{C}_{1}$ where $\hat{C}_{0}$ is the proper transform of $C_{0}$. Let $\hat{f}: \hat{X} \rightarrow \mathbb{P}^{1}$ be the $\mathbb{P}^{1}$-fibration on $\bar{X}$ associated with 
$\hat{C}_{0}$ such that $\hat{C}_{0}=\hat{f}^{-1}(1)$. Consider the fibers $\hat{F}_{1} \cup \hat{F}_{2}$ of $\left.\hat{f}\right|_{X}$ similar to $F_{1} \cup F_{2}$, i.e., every point that is not of type $\hat{F}_{1} \cap \hat{F}_{2}$ belongs to the open orbit $U$ of the $\operatorname{AAut}_{\text {hol }}(X)$ action. Note that by construction the proper transform $G_{i}$ of $F_{i}$ meets $\hat{C}_{0}$ transversely at one point. Hence, its intersection with every other fiber of $\hat{f}$ is also 1 . In particular, $G_{i}$ cannot meet the fiber $\hat{F}_{1} \cup \hat{F}_{2}$ at the double point $\hat{F}_{1} \cap \hat{F}_{2}$. This implies that $G_{1} \cap G_{2} \neq \hat{F}_{1} \cap \hat{F}_{2}$. Thus, $F_{1} \cap F_{2} \in U$ and we are done.

Recall that there are Gizatullin surfaces that are not homogeneous with respect to the natural Aut-action. A list of such surfaces appeared in [26] and we show that every

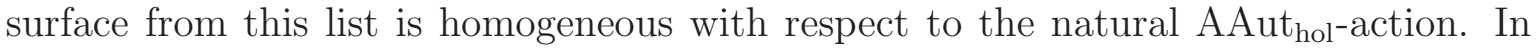
fact this is true for a wider collection of Gizatullin surfaces to describe which we need to remind the following.

Let $\bar{Y}$ be an SNC-completion of a smooth Gizatullin surface $Y$ by a standard zigzag $\bar{D}=\bar{Y} \backslash Y=C_{0} \cup \ldots \cup C_{n-1}, n \geq 3$. The 0 -vertices $C_{0}$ and $C_{1}$ of the zigzag induce two $\mathbb{P}^{1}$-fibrations that lead to a morphism $\bar{\Phi}=\left(\bar{\varphi}_{0}, \bar{\varphi}_{1}\right): \bar{Y} \rightarrow \mathbb{P}^{1} \times \mathbb{P}^{1}$ with restriction $\Phi=\left(\varphi_{0}, \varphi_{1}\right): Y \rightarrow \mathbb{C}_{x, y}^{2}$. Omitting a simple case of $n=3$ we suppose further that $\bar{\Phi}\left(C_{3} \cup \ldots \cup C_{n-1}\right)=(0,0)$, i.e., the only singular fiber $\bar{\varphi}_{0}^{-1}(0)$ of $\bar{\varphi}_{0}$ is contracted by $\bar{\Phi}$ to the proper transform of $C_{2}$. The components of $\bar{\varphi}_{0}^{-1}(0)$ different from $C_{2} \ldots, C_{n-1}$ are called feathers (in terminology of [12] or [26]). For every surface in Kovalenko's list each feather is a $(-1)$-curve.

Theorem 10.3. Let $Y$ be a smooth Gizatullin surface $Y$ such that every feather is a $(-1)$-curve. Then $Y$ is homogeneous with respect to the natural AAuthol-action.

Proof. Since each feather is a $(-1)$-curve they can be contracted first. This implies that for the sequence $\bar{\Phi}: \bar{Y} \rightarrow \mathbb{P}^{1} \times \mathbb{P}^{1}$ of monoidal transformations, $C_{n-1}$ is obtained from the proper transform $0 \times \mathbb{P}^{1}$ of $C_{2}$ after several (say $k$ ) outer blowing-ups in (see Section 3 for definition of outer blowing up) at the origin and infinitely near points. Hence, for some fixed values $a_{1}, \ldots, a_{k-1} \in \mathbb{C}$ and general $b \in \mathbb{C}$ the proper transform $C$ of the curve $y=a_{1} x+\ldots+a_{k-1} x^{k-1}+b x^{k}=: h(x)$ in $\bar{Y}$ meets $C_{n-1}$ at a general point. The triangular automorphism $(x, y) \rightarrow(x, y-h(x))$ of $\mathbb{C}^{2}$ induces an isomorphism of $Y$ on another Gizatullin surface $Y^{\prime}$ which has a completion $\bar{Y}^{\prime}$ by a standard zigzag $C_{0}^{\prime}+\ldots+C_{n-1}^{\prime}$ such that this isomorphism extends regularly to $\bar{Y} \backslash C_{0} \rightarrow \bar{Y}^{\prime} \backslash C_{0}^{\prime}$. We replace $\bar{Y}$ by $\bar{Y}^{\prime}$. The advantage is that $C$ is now the proper transform of the $x$-axis in $\mathbb{C}^{2}$, i.e., it meets both $C_{n-1}$ and $C_{0}$. That is, the graph of $\bar{D} \cup C$ becomes circular with $C$ playing the role of $C_{n}$. Thus, $X=Y \backslash C$ is a surface of type (2) from Theorem 1.4 and by Theorem 10.2 $\mathrm{AAut}_{\text {hol }}(X)$ acts transitively on $X$.

Recall that the field $\nu$ in Case 1 of Theorem 10.2 extends regularly to $\bar{X} \backslash C_{0}$ and in particular to $C_{n} \backslash C_{0}$ (and, therefore, to $Y$ ). Furthermore, consider transformations $\left(\left(0,0, w_{3}, \ldots, w_{n}\right)\right) \rightarrow\left(\left(w_{3}, \ldots, w_{j-2}, 0,0, w_{j}, \ldots, w_{n}\right)\right)$ from Proposition 3.3 used in Case 1 to make a feather adjacent to $C_{2}$ instead of $C_{j}$. Note that $C_{n}$ survives such a transformation and plays the role of $C_{n-j+3}$ in the modified graph, i.e., it is still contained in $\bar{X} \backslash C_{0}$. Hence, even after these transformations the flow of $\nu$ is extendable to $Y$. Since the homogeneity of $X$ is provided by elements of these flows we see that $X$ is contained actually in the open orbit of the $\mathrm{AAut}_{\text {hol }}(Y)$-action. Note that $C \cap Y$ does not contain fixed points of the $\operatorname{AAut}_{\text {hol }}(Y)$-action since each point of $C \cap Y$ can be moved 
by a $\mathbb{G}_{a}$-action induced on $Y$ by the field of form $x^{m} \frac{\partial}{\partial y}$ on $\mathbb{C}^{2}$ with $m>>0$. Thus, the open orbit coincides with $Y$. Therefore, $Y$ is, indeed, $\operatorname{AAut}_{\text {hol }}(Y)$-homogeneous.

\section{REFERENCES}

[1] E. Andersén, Complete vector fields on $\left(\mathbb{C}^{*}\right)^{n}$, Proc. Amer. Math. Soc. 128 (2000), no. 4, 1079-1085.

[2] R. Andrist, Density property for Gizatullin surfaces with reduced degenerate fiber, J. Geom. Anal. 28 (2018), no. 3, 2522-2538.

[3] R. Andrist, F. Kutzschebauch, P.-M. Poloni, Density property for Gizatullin surfaces completed by four rational curves, Proc. Amer. Math. Soc. 145 (2017), no. 12, 5097-5108

[4] W. P. Barth, C. Peters, and A. Van de Ven, Compact Complex Surfaces, Springer 1984.

[5] M. Brunella, Sur les courbes intgrales propres des champs de vecteurs polynomiaux, Topology 37 (1998), no. 6, 1229-1246.

[6] M. Brunella, Birational geometry of foliations, Monografas de Matematica. [Mathematical Monographs] Instituto de Matematica Pura e Aplicada (IMPA), Rio de Janeiro, 2000. 138 pp.

[7] M. Brunella, Foliations on complex projective surfaces, arXiv:math/0212082, (2002), 31 p.

[8] M. Brunella, Complete polynomial vector fields on the complex plane, Topology 43 (2004), 433-455.

[9] D. Daigle, Birational endomorphisms of the affine plane, J. Math. Kyoto Univ. 31 (1991), 329-358.

[10] D. Daigle, Classification of weighted graphs up to blowing-up and blowing-down, Canad. J. Math. 60 (2008), no. 1, 64-87.

[11] H. Flenner, Sh. Kaliman, M. Zaidenberg, Birational transformations of weighted graphs. Affine algebraic geometry, 107-147, Osaka Univ. Press, Osaka, 2007.

[12] H. Flenner, Sh. Kaliman, M. Zaidenberg, Completions of $\mathbb{C}^{*}$-surfaces, Affine algebraic geometry, 149-201, Osaka Univ. Press, Osaka, 2007.

[13] H. Flenner, Sh. Kaliman, M. Zaidenberg, Corrigendum to our paper: Birational transformations of weighted graphs, Affine algebraic geometry, 3538, CRM Proc. Lecture Notes, 54, Amer. Math. Soc., Providence, RI, 2011.

[14] H. Flenner, M. Zaidenberg, Normal affine surfaces with $\mathbb{C}^{*}$-actions, Osaka J. Math. 40 (2003), no. 4, 981-1009.

[15] F. Forstnerič, Stein manifolds and holomorphic mappings Springer, Heidelberg, 2011.

[16] M. Gizatullin, Quasihomogeneous affine surfaces. (Russian) Izv. Akad. Nauk SSSR Ser. Mat. 35 1971 1047-1071.

[17] M. Gizatullin, V. Danilov, Examples of nonhomogeneous quasihomogeneous surfaces. (Russian) Izv. Akad. Nauk SSSR Ser. Mat. 38 (1974), 42-58.

[18] M. Gizatullin, V. Danilov, Automorphisms of affine surfaces. I. (Russian) Izv. Akad. Nauk SSSR Ser. Mat. 39 (1975), no. 3, 523-565, 703.

[19] J. E. Goodman, Affine open subsets of algebraic varieties and ample divisors. Ann. of Math. (2) 89 (1969) 160-183.

[20] A. Guillot, J. Rebelo, Semicomplete meromorphic vector fields on complex surfaces, J. Reine Angew. Math. 667 (2012), 27-65.

[21] Ph. Griffiths, J. Harris, Principles of algebraic geometry, Pure and Applied Mathematics, Wiley-Interscience [John Wiley and Sons], New York, 1978.

[22] R. Hartshorne, Algebraic Geometry, Springer-Verlag, New York Berlin Heidelberg London, 1977, $496 \mathrm{p}$.

[23] Sh. Kaliman, F. Kutzschebauch, On the present state of the Andersen-Lempert theory, In: Affine Algebraic Geometry: The Russell Festschrift, 85-122. Centre de Recherches Mathématiques. CRM Proceedings and Lecture Notes 54, 2011.

[24] Sh. Kaliman, F. Kutzschebauch, On algebraic volume density property, Transform. Groups 21 (2016), no. 2, 451-478. 
[25] Sh. Kaliman, Surfaces with big automorphism groups, Functional Analysis and Geometry: Selim Grigorievich Krein Centennial, Contemporary Mathematics Series, Ed. Peter Kuchment and Evgeny Semenov, 185-196, 2019.

[26] S. Kovalenko, Transitivity of automorphism groups of Gizatullin surfaces, Transitivity of automorphism groups of Gizatullin surfaces. Int. Math. Res. Not. IMRN 2015, no. 21, 11433-11484.

[27] F. Kutzschebauch, M. Leuenberger, A. Liendo, The algebraic density property for affine toric varieties, J. Pure Appl. Algebra 219 (2015), no. 8, 3685-3700.

[28] M. Leuenberger, Complete algebraic vector fields on Danielewski surfaces, Ann. Inst. Fourier (Grenoble) 66 (2016), no. 2, 433-454.

[29] J. Lipman, Desingularization of two-dimensional schemes, Ann. Math. (2) 107:1 (1978), 151207.

[30] D. Luna, Slices tales, (French) Sur les groupes algbriques, pp. 81-105. Bull. Soc. Math. France, Paris, Memoire 33 Soc. Math. France, Paris, 1973.

[31] M. McQuillan, Canonical models of foliations, Pure Appl. Math. Q. 4 (2008), no. 3, part 2, 877-.-1012.

[32] M. McQuillan, Non-commutative Mori Theory, IHES, M/01/42, (2001).

[33] M. Miyanishi, Open algebraic surfaces, CRM Monograph Series, 12. American Mathematical Society, Providence, RI, 2001.

[34] M. Miyanishi, Singularities of normal affine surfaces containing cylinderlike open sets, J. Algebra 68 (1981), no. 2, 268-275.

[35] P. Orlik, P. Wagreich, Isolated singularities of algebraic surfaces with $\mathbb{C}^{*}$ action, Ann. Math. 93 (1971), 205-228.

[36] H. Pinkham, Normal surface singularities with $\mathbb{C}^{*}$-action. Math. Ann. 227 (1977), no. 2, 183193.

[37] M. Schütt, T. Shioda, Elliptic surfaces. Algebraic geometry in East Asia - Seoul 2008, 51-160, Adv. Stud. Pure Math., 60, Math. Soc. Japan, Tokyo, 2010.

[38] P. Scott, The geometries of 3-manifolds, Bull. London Math. Soc. 15 (1983), no. 5, 401-487.

[39] A. Seidenberg, Reduction of singularities of the differential equation $A d y=B d$, Amer. J. Math. 89 (1968), 248-269.

[40] M. Suzuki, Sur les opérations holomorphes du groupe additif complexe sur l'espace de deux variables complexes, Ann. Sci. École Norm. Sup. 10 (1977), no. 4, 517-546.

[41] M. Suzuki, Sur les opérations holomorphes de $\mathbb{C}$ et de $\mathbb{C}^{*}$ sur un espace de Stein, Fonctions de plusieurs variables complexes, III (Sém. Francois Norguet, 1975-1977), pp. 80-88, 394, Lecture Notes in Math., 670, Springer, Berlin, 1978.

[42] M. Suzuki, Sur les intégrales premières de certains feuilletages analytiques complexes, (French) Fonctions de plusieurs variables complexes, III (Sém. Francois Norguet, 1975-1977), pp. 53-79, 394, Lecture Notes in Math., 670, Springer, Berlin, 1978.

Department of Mathematics, University of Miami, Coral Gables, FL 33124, USA

E-mail address: kaliman@math.miami.edu

Department of Mathematics, University of Bern, Bern, Switzerland,

E-mail address: frank.kutzschebauch@math.unibe.ch

Department of Mathematics, University of Bern, Bern, Switzerland, E-mail address: matthias.leuenberger@math.unibe.ch 